\title{
Existence uniqueness and stability of mild solutions for semilinear $\psi$-Caputo fractional evolution equations
}

\author{
Apassara Suechoei ${ }^{1}$ and Parinya Sa Ngiamsunthorn ${ }^{1 *}$
}

\section{"Correspondence:}

parinya.san@kmutt.ac.th

'Department of Mathematics,

Faculty of Science, King Mongkut's

University of Technology Thonburi,

Bangkok, Thailand

\begin{abstract}
In this paper, we study the local and global existence, and uniqueness of mild solution to initial value problems for fractional semilinear evolution equations with compact and noncompact semigroup in Banach spaces. In particular, we derive the form of fundamental solution in terms of semigroup induced by resolvent and $\psi$-function from Caputo fractional derivatives. These results generalize previous work where the classical Caputo fractional derivative is considered. Moreover, we prove the Mittag-Leffler-Ulam-Hyers stability result. Finally, we give examples of time-fractional heat equation to illustrate the result.
\end{abstract}

MSC: Primary 34A08; secondary 35A01; 35A02

Keywords: Fractional evolution equation; Existence and uniqueness theorems; Mild solution; Measure of noncompactness; Mittag-Leffler-Ulam-Hyers stability

\section{Introduction}

Fractional differential equations have been applied in many fields, such as economics, engineering, chemistry, physics, finance, aerodynamics, electrodynamics of complex medium, polymer rheology, control of dynamical systems (see [1-16]). The research on fractional calculus has become a focus area of study due to the fact that some dynamical models can be described more accurately with fractional derivatives than the ones with integer-order derivatives. In particular, it is shown that fractional calculus provides more realistic models demonstrating hidden aspects in a model of spring pendulum [13], the free motion of a particle in a circular cavity [11] and some epidemic models [15, 17].

Several researchers are interested in investigating various aspects of fractional differential equations such as existence and uniqueness of solutions, exact solutions, stability of solutions, and methods for explicit and numerical solutions [17-20]. The common techniques used to display the existence and uniqueness of solutions are fixed point theorem, upper-lower solutions, iterative method and numerical method. For stability of solutions, there is a concept of data dependence, which becomes one of significant topics in the analysis of fractional differential equations, called the Ulam-Hyers stability (see [21-23]).

(c) The Author(s) 2020. This article is licensed under a Creative Commons Attribution 4.0 International License, which permits use sharing, adaptation, distribution and reproduction in any medium or format, as long as you give appropriate credit to the original author(s) and the source, provide a link to the Creative Commons licence, and indicate if changes were made. The images or other third party material in this article are included in the article's Creative Commons licence, unless indicated otherwise in a credit line to the material. If material is not included in the article's Creative Commons licence and your intended use is not permitted by statutory regulation or exceeds the permitted use, you will need to obtain permission directly from the copyright holder. To view a copy of this licence, visit http://creativecommons.org/licenses/by/4.0/. 
One of the main research focuses on fractional calculus is the theory of fractional evolution equations since they are abstract formulation for many problems arising in engineering and physics. Evolution equations are commonly used to describe the systems that change or evolve over time. A number of studies have been conducted on the existence and unique of solutions for fractional evolution equations based on semigroup and fixed point theory (see [24-32]). On the other hand, there has been some studies about fundamental solution for homogeneous fractional evolution equations [33, 34]. Recently, [19] applied the homotopy analysis transform method (HATM) for solving time-fractional Cauchy reaction-diffusion equations. In addition, Wang and Zhou [35] presented four kind of stabilities of the mild solution of the fractional evolution equation in Banach space, namely Mittag-Leffler-Ulam-Hyers stability, generalized MittagLeffler-Ulam-Hyers stability, Mittag-Leffler-Ulam-Hyers-Rassias stability and generalized Mittag-Leffler-Ulam-Hyers-Rassias stability.

There is variation in the definition of fractional differential operators found in the literature, including Riemann-Liouville, Caputo, Hilfer, Riesz, Erdelyi-Kober, and Hadamard $[2,36]$ operators. The common definitions that triggered attention from many researchers are Riemann-Liouville and Caputo fractional calculus. In Riemann-Liouville fractional differential modeling, the initial condition involves limit values of fractional derivatives, which is difficult to interpret. The Caputo fractional derivative has the advantage of being suitable for physical models with initial condition because the physical interpretation of the prescribed data is clear and it is in general possible to provide these data by suitable measurements [37].

Almeida [38] generalized the definition of Caputo fractional derivative by considering the Caputo fractional derivative of a function with respect to another function $\psi$ and studied some useful properties of the fractional calculus. The advantage of this new definition of the fractional derivative is that a higher accuracy of the model could be achieved by choosing a suitable function $\psi$.

Recently, Jarad and Abdeljawad [39] introduced the generalized Laplace transform with respect to another function and the inverse version of the Laplace transform with respect to another function. This can be used to solve some fractional differential equations in the framework of generalized Caputo fractional derivative.

Motivated by the work of $[25,39]$, we consider the following fractional evolution equation in a Banach space $E$ :

$$
\left\{\begin{array}{l}
{ }_{0}^{C} D_{\psi}^{\alpha} u(t)=\mathcal{A} u(t)+f(t, u(t)), \quad t \in(0, T] \\
u(0)=u_{0}
\end{array}\right.
$$

where $0<\alpha<1, T<\infty, \mathcal{A}$ is the infinitesimal generator of a $C_{0}$-semigroup of uniformly bounded linear operators $\{T(t)\}_{t \geq 0}$ on $E, u_{0} \in E$ and $f:[0, \infty) \times E \rightarrow E$ is given function. The fractional derivative ${ }_{0}^{\mathrm{C}} D_{\psi}^{\alpha}$ considered in this work is in the sense of Caputo fractional derivative with respect to a function $\psi$ which gives a more general framework to the results in the literature. Moreover, this problem is more general than the work in [39] where we consider the evolution operator $\mathcal{A}$ instead of a constant.

In this paper, we aim to establish a mild solution for the problem (1) in terms of semigroup depending on a function $\psi$ from the generalized Caputo derivative. In addition, we prove the existence and uniqueness results of mild solution for the problem (1) in local and 
global time under the condition that $\{T(t)\}_{t \geq 0}$ is both compact and noncompact operator. The results obtained in this work are in the abstract form which can be applied for further investigation such as the evolution equations with perturbation, delay and nonlocal term.

This paper will be organized as follows. In Sect. 2, we will briefly recall some basic definitions and some preliminary concepts about fractional calculus and auxiliary results used in the following sections. We then construct a mild solution by using semigroup for the problem in Sect. 3. We prove the existence and uniqueness of mild solutions of the problem (1) under compact and noncompact analytic semigroup by the Schauder fixed point theorem in Sects. 4 and 5, respectively. In Sect. 6 we present Mittag-Leffler-Ulam-Hyers stability result for the problem (1). Finally, we give some examples to illustrate the application of the results obtained in Sect. 7 and our conclusion in Sect. 8.

\section{Preliminaries}

In this section, we introduce preliminary background which is used throughout this paper.

Let $E$ be a Banach space with the norm $\|\cdot\|$ and let $C(J, E)$ be the Banach space of continuous functions from $J$ to $E$ with the norm $\|u\|_{C}=\sup _{t \in J}\|u(t)\|$.

Definition 2.1 ( $\psi$-Riemann-Liouville fractional integral [39]) Let $\alpha>0, f$ be an integrable function defined on $[a, b]$ and $\psi \in C^{1}([a, b])$ be an increasing function with $\psi^{\prime}(t) \neq 0$ for all $t \in[a, b]$. The $\psi$-Riemann-Liouville fractional integral operator of order $\alpha$ of a function $f$ is defined by

$$
\left({ }_{a} I_{\psi}^{\alpha} f\right)(t)=\frac{1}{\Gamma(\alpha)} \int_{a}^{t}(\psi(t)-\psi(s))^{\alpha-1} f(s) \psi^{\prime}(s) d s
$$

It is obvious that when $\psi(t)=t,(2)$ is the classical Riemann-Liouville's fractional integral.

Definition 2.2 ( $\psi$-Riemann-Liouville fractional derivative [39]) Let $n-1<\alpha<n, f$ be an integrable function defined on $[a, b]$ and $\psi \in C^{1}([a, b])$ be an increasing function with $\psi^{\prime}(t) \neq 0$ for all $t \in[a, b]$. The $\psi$-Riemann-Liouville fractional derivative of order $\alpha$ of a function $f$ is defined by

$$
\begin{aligned}
\left({ }_{a} D_{\psi}^{\alpha} f\right)(t) & =\left(\frac{1}{\psi^{\prime}(t)} \frac{d}{d t}\right)^{n}\left({ }_{a} I_{\psi}^{n-\alpha} f\right)(t) \\
& =\frac{\left(\frac{1}{\psi^{\prime}(t)} \frac{d}{d t}\right)^{n}}{\Gamma(n-\alpha)} \int_{a}^{t}(\psi(t)-\psi(s))^{n-\alpha-1} f(s) d s
\end{aligned}
$$

where $n=[\alpha]+1$.

From the definition, when $\alpha=n \in \mathbb{N}$, we have

$$
\left({ }_{a} D_{\psi}^{\alpha} f\right)(t)=\left(\frac{1}{\psi^{\prime}(t)} \frac{d}{d t}\right)^{n} f(t)
$$

Definition 2.3 Let $\psi \in C^{n}([a, b])$ be such that $\psi^{\prime}(t)>0$ on $[a, b]$. Then

$$
A C_{\psi}^{n}([a, b])=\left\{f:[a, b] \rightarrow \mathbb{C} \text { and } f^{[n-1]}=\left(\frac{1}{\psi^{\prime}(t)} \frac{d}{d t}\right)^{n-1} f\right\}
$$


Lemma 2.4 ([39, 40]) Let $\alpha>0$ and $\beta>0$, then

(i) ${ }_{a} I_{\psi}^{\alpha}(\psi(x)-\psi(a))^{\beta-1}(t)=\frac{\Gamma(\beta)}{\Gamma(\beta+\alpha)}(\psi(t)-\psi(a))^{\beta+\alpha-1}$;

(ii) ${ }_{a} D_{\psi}^{\alpha}(\psi(x)-\psi(a))^{\beta-1}(t)=\frac{\Gamma(\beta)}{\Gamma(\beta-\alpha)}(\psi(t)-\psi(a))^{\beta+\alpha-1}$.

Definition 2.5 ( $\psi$-Caputo fractional derivative $[38,39])$ Let $n-1<\alpha<n, f \in C^{n}([a, b])$ and $\psi \in C^{n}([a, b])$ be an increasing function with $\psi^{\prime}(t) \neq 0$ for all $t \in[a, b]$. The $\psi$-Caputo fractional derivative of order $\alpha$ of a function $f$ is defined by

$$
\begin{aligned}
\left({ }_{a}^{\mathrm{C}} D_{\psi}^{\alpha} f\right)(t) & =\left({ }_{a} I_{\psi}^{n-\alpha} f^{[n]}\right)(t) \\
& =\frac{1}{\Gamma(n-\alpha)} \int_{a}^{t}(\psi(t)-\psi(s))^{n-\alpha-1} f^{[n]}(s) \psi^{\prime}(s) d u,
\end{aligned}
$$

where $n=[\alpha]+1$ and $f^{[n]}(t):=\left(\frac{1}{\psi^{\prime}(t)} \frac{d}{d t}\right)^{n} f(t)$ on $[a, b]$.

From the definition, it is clear that, when $\alpha=n \in \mathbb{N}$,

$$
\left({ }_{a}^{C} D_{\psi}^{\alpha} f\right)(t)=f^{[n]}(t) .
$$

Remark $2.6([38,39])$ The relationship between the $\psi$-Caputo and the $\psi$-RiemannLiouville derivatives can be written as follows:

$$
\begin{aligned}
\left({ }_{a}^{\mathrm{C}} D_{\psi}^{\alpha} f\right)(t) & =\left({ }_{a} D_{\psi}^{\alpha} f\right)(t)-\sum_{k=0}^{n-1} \frac{f^{[k]}\left(a^{+}\right)}{\Gamma(k-\alpha+1)}(\psi(t)-\psi(a))^{k-\alpha} \\
& ={ }_{a} D_{\psi}^{\alpha}\left(f(s)-\sum_{k=0}^{n-1} \frac{f^{[k]}\left(a^{+}\right)}{k !}(\psi(s)-\psi(a))^{k}\right)(t),
\end{aligned}
$$

where $t>a$ and $n=[\alpha]+1$.

Theorem 2.7 ([38]) Let $f \in C^{n}([a, b])$ and $\alpha>0$. Then we have

$$
{ }_{a} I_{\psi}^{\alpha}{ }_{a}^{C} D_{\psi}^{\alpha} f(t)=f(t)-\sum_{k=0}^{n-1} \frac{f^{[k]}\left(a^{+}\right)}{k !}(\psi(t)-\psi(a))^{k} .
$$

In particular, given $\alpha \in(0,1)$, we have

$$
{ }_{a} I_{\psi a}^{\alpha}{ }_{a}^{C} D_{\psi}^{\alpha} f(t)=f(t)-f(a) .
$$

Definition 2.8 ([39]) Let $u, \psi:[a, \infty) \rightarrow \mathbb{R}$ be real valued functions such that $\psi(t)$ is continuous and $\psi^{\prime}(t)>0$ on $[0, \infty)$. The generalized Laplace transform of $f$ is denoted by

$$
\mathcal{L}_{\psi}\{u(t)\}(s)=\int_{a}^{\infty} e^{-s(\psi(t)-\psi(a))} u(t) \psi^{\prime}(t) d t
$$

for all $s$.

Definition 2.9 ([39]) Let $u$ and $v$ be two functions which are piecewise continuous at each interval $[0, T]$ and of exponential order. We define the generalized convolution of $u$ and $v$ 
by

$$
\left(u *_{\psi} v\right)(t)=\int_{a}^{t} u(\tau) v\left(\psi^{-1}(\psi(t)+\psi(a)-\psi(\tau))\right) \psi^{\prime}(\tau) d \tau .
$$

Theorem 2.10 ([39]) Let $\alpha>0$ and $f$ be a piecewise continuous function on each interval $[a, t]$ and $\psi(t)$-exponential order. Then

$$
\mathcal{L}_{\psi}\left\{{ }_{a} I_{\psi}^{\alpha} u(t)\right\}(s)=\frac{{ }_{a} I_{\psi}^{\alpha} u(t)}{s^{\alpha}} .
$$

Theorem 2.11 (Gronwall's inequality $[41,42]$ ) Let $u, v$ be two integrable functions and $h$ be a continuous function on $[a, b]$. Let $\psi \in C^{1}([a, b])$ be an increasing function such that $\psi^{\prime}(t) \neq 0$ for all $t \in[a, b]$. Assume that

(1) $u$ and $v$ are nonnegative;

(2) $h$ is nonnegative and nondecreasing.

If

$$
u(t) \leq v(t)+h(t) \int_{a}^{t}(\psi(t)-\psi(s))^{\alpha-1} u(s) \psi^{\prime}(s) d s,
$$

then

$$
u(t) \leq v(t)+\int_{a}^{t} \sum_{k=1}^{\infty} \frac{[h(t) \Gamma(\alpha)]^{k}}{\Gamma(n \alpha)}(\psi(t)-\psi(s))^{k \alpha-1} v(s) \psi^{\prime}(s) d s
$$

for all $t \in[a, b]$.

Corollary 2.12 Under the hypotheses of Theorem 2.11, let $v$ be a nondecreassing function on $[a, b]$. Then we have

$$
u(t) \leq v(t) E_{\alpha}\left(h(t) \Gamma(\alpha)[\psi(t)-\psi(a)]^{\alpha}\right)
$$

for all $t \in[a, b]$, where $E_{\alpha}(z)=\sum_{k=0}^{\infty} \frac{z^{k}}{\Gamma(k \alpha+1)}$ is the Mittag-Leffler function with one parameter for $z \in \mathbb{C}$ and $\alpha>0$.

Definition $2.13([43,44])$ The Wright type function is given by

$$
\begin{aligned}
\phi_{\alpha}(z) & =\sum_{k=0}^{\infty} \frac{(-z)^{k}}{k ! \Gamma(-\alpha k+1-\alpha)} \\
& =\sum_{k=0}^{\infty} \frac{(-z)^{k} \Gamma(\alpha(k+1)) \sin (\pi(k+1) \alpha)}{k !}
\end{aligned}
$$

for $0<\alpha<1$ and $z \in \mathbb{C}$.

Proposition 2.14 $([43,44])$ The Wright function $\phi_{\alpha}$ is an entire function and has the following properties:

(i) $\phi_{\alpha}(\theta) \geq 0$ for $\theta \geq 0$ and $\int_{0}^{\infty} \phi_{\alpha}(\theta) d \theta=1$; 

(ii) $\int_{0}^{\infty} \phi_{\alpha}(\theta) \theta^{r} d \theta=\frac{\Gamma(1+r)}{\Gamma(1+\alpha r)}$ for $r>-1$;
(iii) $\int_{0}^{\infty} \phi_{\alpha}(\theta) e^{-z \theta} d \theta=E_{\alpha}(-z), \quad z \in \mathbb{C}$;
(iv) $\alpha \int_{0}^{\infty} \theta \phi_{\alpha}(\theta) e^{-z \theta} d \theta=E_{\alpha, \alpha}(-z), \quad z \in \mathbb{C}$.

Next, we introduce the definition for Kuratowski measure of noncompactness, which will be used in the proof of our main results.

Definition 2.15 ([45]) Let $E$ be a Banach space and $\mathcal{B}(E)$ be the bounded subset of $E$. The Kuratowski measure of noncompactness is the map $\mu: \mathcal{B}(E) \rightarrow[0, \infty)$ define by

$$
\mu(B)=\inf \left\{\varepsilon>0: B \subset \bigcup_{j=1}^{\infty} B_{j}, \operatorname{diam}\left(B_{j}\right)<\varepsilon \text { for } i=1,2, \ldots, n\right\}
$$

where $\operatorname{diam}\left(B_{j}\right)=\sup \left\{|x-y|: x, y \in B_{j}\right\}$.

The following properties of the Kuratowski measure of noncompactness are well known.

Lemma $2.16([45,46])$ Let $E$ be Banach spaces and $U, V \subset E$ be bounded. Then the noncompactness measure has the following properties:

(i) $\mu(U)=0$ if and only if $\bar{U}$ is compact, where $\bar{U}$ means the closure hull of $U$;

(ii) $\mu(\lambda U)=|\lambda| \mu(U)$, where $\lambda \in \mathbb{R}$;

(iii) $\mu(U)=\mu(\bar{U})=\mu(\operatorname{conv} U)$, where conv $U$ means the convex hull of $U$;

(iv) $\mu(U \cup V)=\max \{\mu(U), \mu(V)\}$;

(v) $\mu(U) \leq \mu(V)$ if $U \subset V$;

(vi) $\mu(U+V) \leq \mu(U)+\mu(V)$; where $U+V=\{x \mid x=y+z, y \in U, z \in V\}$;

(vii) $\mu(U+x)=\mu(U)$, for any $x \in E$;

(viii) If the map $Q: \operatorname{dom}(Q) \subset E \rightarrow X$ is Lipschitz continuous with constant $k$, then $\mu(Q(S)) \leq k \mu(S)$ for any bounded subset $S \subset \operatorname{dom}(Q)$, where $X$ is another Banach space.

Lemma 2.17 ([47]) Let E be a Banach space, and let $D \subset E$ be bounded. Then there exists a countable set $D_{0} \subset D$ such that $\mu(D) \leq 2 \mu\left(D_{0}\right)$.

Lemma $2.18([45,46])$ Let $E$ be a Banach space, and $B \subset C(J, E), B(t)=\{u(t): u \in B\}$ $(t \in J)$. If $B$ is bounded and equicontinuous, then $\mu(B(t))$ is continuous on $J$, and $\mu(B)=$ $\max _{t \in J} \mu(B(t))=\mu(B(J))$.

Lemma 2.19 ([48]) Let E be a Banach space, and let $B \subset C(J, E)$ be bounded and equicontinuous. Then $\mu(B(t))$ is continuous on J, and

$$
\mu\left(\left\{\int_{J} u(t) d t \mid u \in B\right\}\right) \leq \int_{J} \mu(B(t)) d t
$$


Lemma 2.20 ([49]) Let E be a Banach space, and let $B=\left\{u_{n}\right\}_{n=1}^{\infty} \subset C(J, E)$ be a bounded and countable set. Then $\mu(B(t))$ is Lebesgue integrable on $J$, and

$$
\mu\left(\left\{\int_{J} u_{n}(t) d t\right\}_{n=1}^{\infty}\right) \leq 2 \int_{J} \mu(B(t)) d t
$$

Lemma 2.21 ([50]) If $B \subset C(J, E)$ is bounded and equicontinuous, then $\overline{\mathrm{Co}} B \subset C(J, E)$ is also bounded and equicontinuous.

Lemma 2.22 ([45]) Let E be a Banach space. Assume that $D \subset E$ is a bounded closed and convex set on $E, Q: D \rightarrow D$ is condensing. Then $Q$ has at least one fixed point in $D$.

Lemma 2.23 (Schauder's fixed point theorem) Let $E$ be a Banach space and $D \subset E, a$ convex, closed and bounded set. If $T: D \rightarrow D$ is a continuous operator such that $T(D) \subset E$, $T(D)$ is relatively compact, then $T$ has at least one fixed point in $D$.

Next, we give some facts about the semigroups of linear operators. These results can be found in [51, 52].

For a strongly continuous semigroup (i.e., $C_{0}$-semigroup) $\{T(t)\}_{t \geq 0}$, the infinitesimal generator of $\{T(t)\}_{t \geq 0}$ is defined by

$$
\mathcal{A} x=\lim _{t \rightarrow 0^{+}} \frac{T(t) x-x}{x}, \quad x \in E .
$$

We denote by $D(\mathcal{A})$ the domain of $\mathcal{A}$, that is,

$$
D(\mathcal{A})=\left\{x \in E: \lim _{t \rightarrow 0^{+}} \frac{T(t) x-x}{x} \text { exists }\right\} .
$$

Lemma 2.24 ([51,52]) Let $\{T(t)\}_{t \geq 0}$ be a $C_{0}$-semigroup, then there exist constants $C \geq 1$ and $a \geq 0$ such that $\|T(t)\| \leq C e^{a t}$ for all $t \geq 0$.

Lemma $2.25([51,52])$ A linear operator $\mathcal{A}$ is the infinitesimal generator of a $C_{0}$-semigroup if and only if

(i) $\mathcal{A}$ is closed and $\overline{D(\mathcal{A})}=E$.

(ii) The resolvent set $\rho(\mathcal{A})$ of $\mathcal{A}$ contains $\mathbb{R}^{+}$and, for every $\lambda>0$, we have

$$
\|R(\lambda, \mathcal{A})\| \leq \frac{1}{\lambda}
$$

where $R(\lambda, \mathcal{A}):=\left(\lambda^{\alpha} I-\mathcal{A}\right)^{-1} x=\int_{0}^{\infty} e^{-\lambda^{\alpha} t} T(t) x d t$.

Throughout this paper, let $\mathcal{A}$ be the infinitesimal generator of a $C_{0}$-semigroup of uniformly bounded linear operators $\{T(t)\}_{t \geq 0}$ on $E$. Then there exists $M \geq 1$ such that $M=\sup _{t \in[0, \infty)}\|T(t)\|$. 


\section{Representation of mild solution using semigroup}

According to Definition 2.5 and Theorem 2.7, it is suitable to rewrite the Cauchy problem in the equivalent integral equation

$$
u(t)=u_{0}+\frac{1}{\Gamma(\alpha)} \int_{0}^{t}(\psi(t)-\psi(\tau))^{\alpha-1}(\mathcal{A} u(\tau)+f(\tau, u(\tau))) \psi^{\prime}(\tau) d \tau .
$$

Lemma 3.1 If (8) holds, then we have

$$
\begin{aligned}
u(t)= & \int_{0}^{\infty} \phi_{\alpha}(\theta) T\left((\psi(t)-\psi(0))^{\alpha} \theta\right) u_{0} d \theta \\
& +\alpha \int_{0}^{t} \int_{0}^{\infty} \phi_{\alpha}(\theta)(\psi(t)-\psi(s))^{\alpha-1} T\left((\psi(t)-\psi(0))^{\alpha} \theta\right) f(s, u(s)) \psi^{\prime}(s) d \theta d s .
\end{aligned}
$$

Proof Let $\lambda>0$. Applying the generalized Laplace transforms to (8), we have

$$
U(\lambda)=\frac{u_{0}}{\lambda}+\frac{1}{\lambda^{\alpha}}(\mathcal{A} U(\lambda)+F(\lambda))
$$

where

$$
U(\lambda)=\int_{0}^{\infty} e^{-\lambda(\psi(\tau)-\psi(0))} u(\tau) \psi^{\prime}(\tau) d \tau
$$

and

$$
F(\lambda)=\int_{0}^{\infty} e^{-\lambda(\psi(\tau)-\psi(0))} f(\tau, u(\tau)) \psi^{\prime}(\tau) d \tau
$$

It follows that

$$
\begin{aligned}
U(\lambda) & =\lambda^{\alpha-1}\left(\lambda^{\alpha} I-\mathcal{A}\right)^{-1} u_{0}+\left(\lambda^{\alpha} I-\mathcal{A}\right)^{-1} F(\lambda) \\
& =\lambda^{\alpha-1} \int_{0}^{\infty} e^{-\lambda^{\alpha} s} T(s) u_{0} d s+\int_{0}^{\infty} e^{-\lambda^{\alpha} s} T(s) F(\lambda) d s \\
& =\alpha \int_{0}^{\infty}(\lambda \hat{t})^{\alpha-1} e^{-(\lambda \hat{t})^{\alpha}} T\left(\hat{t}^{\alpha}\right) u_{0} d t+\alpha \int_{0}^{\infty} \hat{t}^{\alpha-1} e^{-(\lambda t)^{\alpha}} T\left(\hat{t}^{\alpha}\right) F(\lambda) d t \\
& =: I_{1}+I_{2} .
\end{aligned}
$$

Taking $\hat{t}=\psi(t)-\psi(0)$, we obtain

$$
\begin{aligned}
I_{1} & =\alpha \int_{0}^{\infty} \lambda^{\alpha-1}(\psi(t)-\psi(0))^{\alpha-1} e^{-(\lambda(\psi(t)-\psi(0)))^{\alpha}} T\left((\psi(t)-\psi(0))^{\alpha}\right) u_{0} \psi^{\prime}(t) d t \\
& =\int_{0}^{\infty}-\frac{1}{\lambda} \frac{d}{d t}\left(e^{-(\lambda(\psi(t)-\psi(0)))^{\alpha}}\right) T\left((\psi(t)-\psi(0))^{\alpha}\right) u_{0} d t
\end{aligned}
$$

and

$$
\begin{aligned}
I_{2}= & \int_{0}^{\infty} \alpha(\psi(t)-\psi(0))^{\alpha-1} e^{-(\lambda(\psi(t)-\psi(0)))^{\alpha}} T\left((\psi(t)-\psi(0))^{\alpha}\right) F(\lambda) \psi^{\prime}(t) d t \\
= & \int_{0}^{\infty} \int_{0}^{\infty} \alpha(\psi(t)-\psi(0))^{\alpha-1} e^{-(\lambda(\psi(t)-\psi(0)))^{\alpha}} T\left((\psi(t)-\psi(0))^{\alpha}\right) \\
& e^{-(\lambda(\psi(s)-\psi(0)))} f(s, u(s)) \psi^{\prime}(s) \psi^{\prime}(t) d s d t .
\end{aligned}
$$


We consider the following one-sided stable probability density in [53]:

$$
\rho_{\alpha}(\theta)=\frac{1}{\pi} \sum_{k=1}^{\infty}(-1)^{k-1} \theta^{-\alpha k-1} \frac{\Gamma(\alpha k+1)}{k !} \sin (k \pi \alpha), \quad \theta \in(0, \infty)
$$

whose integration is given by

$$
\int_{0}^{\infty} e^{-\lambda \theta} \rho_{\alpha}(\theta) d \theta=e^{-\lambda^{\alpha}}, \quad \text { where } \alpha \in(0,1) .
$$

Using (10), we get

$$
\begin{aligned}
& \int_{0}^{\infty}-\frac{1}{\lambda} \frac{d}{d t}\left(e^{-(\lambda(\psi(t)-\psi(0)))^{\alpha}}\right) T\left((\psi(t)-\psi(0))^{\alpha}\right) u_{0} d t \\
& \quad=\int_{0}^{\infty} \int_{0}^{\infty} \theta \rho_{\alpha}(\theta) e^{-\lambda(\psi(t)-\psi(0)) \theta} T\left((\psi(t)-\psi(0))^{\alpha}\right) u_{0} \psi^{\prime}(t) d \theta d t \\
& \quad=\int_{0}^{\infty} e^{-\lambda(\psi(t)-\psi(0))}\left(\int_{0}^{\infty} \rho_{\alpha}(\theta) T\left(\frac{(\psi(t)-\psi(0))^{\alpha}}{\theta^{\alpha}}\right) u_{0} d \theta\right) \psi^{\prime}(t) d t
\end{aligned}
$$

and

$$
\begin{aligned}
& \int_{0}^{\infty} \int_{0}^{\infty} \alpha(\psi(t)-\psi(0))^{\alpha-1} e^{-(\lambda(\psi(t)-\psi(0)))^{\alpha}} T\left((\psi(t)-\psi(0))^{\alpha}\right) e^{-(\lambda(\psi(s)-\psi(0)))} \\
& f(s, u(s)) \psi^{\prime}(s) \psi^{\prime}(t) d s d t \\
&= \int_{0}^{\infty} \int_{0}^{\infty} \int_{0}^{\infty} \alpha(\psi(t)-\psi(0))^{\alpha-1} \rho_{\alpha}(\theta) e^{-\lambda(\psi(t)-\psi(0)) \theta} T\left((\psi(t)-\psi(0))^{\alpha}\right) \\
& e^{-\lambda(\psi(s)-\psi(0))} f(s, u(s)) \psi^{\prime}(s) \psi^{\prime}(t) d \theta d s d t \\
&= \int_{0}^{\infty} \int_{0}^{\infty} \int_{0}^{\infty} \alpha e^{-\lambda(\psi(t)+\psi(s)-2 \psi(0))} \frac{(\psi(t)-\psi(0))^{\alpha-1}}{\theta^{\alpha}} \rho_{\alpha}(\theta) T\left(\frac{(\psi(t)-\psi(0))^{\alpha}}{\theta^{\alpha}}\right) \\
&= \int_{0}^{\infty} \int_{t}^{\infty} \int_{0}^{\infty} \alpha e^{-\lambda(\psi(\tau)-\psi(0))} \rho_{\alpha}(\theta) \frac{(\psi(t)-\psi(0))^{\alpha-1}}{\theta^{\alpha}} T\left(\frac{(\psi(t)-\psi(0))^{\alpha}}{\theta^{\alpha}}\right) \\
& f\left(\psi^{-1}(\psi(\tau)-\psi(t)+\psi(0)), u\left(\psi^{-1}(\psi(\tau)-\psi(t)+\psi(0))\right)\right) \\
& \times \psi^{\prime}(\tau) \psi^{\prime}(t) d \theta d \tau d t \\
&= \int_{0}^{\infty} \int_{0}^{\tau} \int_{0}^{\infty} \alpha e^{-\lambda(\psi(\tau)-\psi(0))} \rho_{\alpha}(\theta) \frac{(\psi(t)-\psi(0))^{\alpha-1}}{\theta^{\alpha}} T\left(\frac{(\psi(t)-\psi(0))^{\alpha}}{\theta^{\alpha}}\right) \\
& f\left(\psi^{-1}(\psi(\tau)-\psi(t)+\psi(0)), u\left(\psi^{-1}(\psi(\tau)-\psi(t)+\psi(0))\right)\right) \\
& \times \psi^{\prime}(\tau) \psi^{\prime}(t) d \theta d t d \tau \\
&= \int_{0}^{\infty} e^{-\lambda(\psi(\tau)-\psi(0))} \\
& \times\left(\int_{0}^{\tau} \int_{0}^{\infty} \alpha \rho_{\alpha}(\theta) \frac{(\psi(\tau)-\psi(s))^{\alpha-1}}{\theta^{\alpha}}\left(\frac{(\psi(\tau)-\psi(s))^{\alpha}}{\theta^{\alpha}}\right) f(s, u(s)) \psi^{\prime}(s) d \theta d s\right) \\
& \times \psi^{\prime}(\tau) d \tau .
\end{aligned}
$$


Then we get

$$
\begin{aligned}
& U(\lambda) \\
& =\int_{0}^{\infty} e^{-\lambda(\psi(t)-\psi(0))}\left(\int_{0}^{\infty} \rho_{\alpha}(\theta) T\left(\frac{(\psi(t)-\psi(0))^{\alpha}}{\theta^{\alpha}}\right) u_{0} d \theta\right) \psi^{\prime}(t) d t \\
& \quad+\int_{0}^{\infty} e^{-\lambda(\psi(\tau)-\psi(0))} \\
& \quad \times\left(\int_{0}^{\tau} \int_{0}^{\infty} \alpha \rho_{\alpha}(\theta) \frac{(\psi(\tau)-\psi(s))^{\alpha-1}}{\theta^{\alpha}} T\left(\frac{(\psi(\tau)-\psi(s))^{\alpha}}{\theta^{\alpha}}\right) f(s, u(s)) \psi^{\prime}(s) d \theta d s\right) \\
& \quad \times \psi^{\prime}(\tau) d \tau .
\end{aligned}
$$

Now, we can invert the Laplace transform to get

$$
\begin{aligned}
u(t)= & \int_{0}^{\infty} \rho_{\alpha}(\theta) T\left(\frac{(\psi(t)-\psi(0))^{\alpha}}{\theta^{\alpha}}\right) u_{0} d \theta \\
& +\alpha \int_{0}^{t} \int_{0}^{\infty} \rho_{\alpha}(\theta) \frac{(\psi(t)-\psi(s))^{\alpha-1}}{\theta^{\alpha}} T\left(\frac{(\psi(t)-\psi(s))^{\alpha}}{\theta^{\alpha}}\right) f(s, u(s)) \psi^{\prime}(s) d \theta d s \\
= & \int_{0}^{\infty} \phi_{\alpha}(\theta) T\left((\psi(t)-\psi(0))^{\alpha} \theta\right) u_{0} d \theta \\
& +\alpha \int_{0}^{t} \int_{0}^{\infty} \theta \phi_{\alpha}(\theta)(\psi(t)-\psi(s))^{\alpha-1} T\left((\psi(t)-\psi(0))^{\alpha} \theta\right) f(s, u(s)) \psi^{\prime}(s) d \theta d s,
\end{aligned}
$$

where $\phi_{\alpha}(\theta)=\frac{1}{\alpha} \theta^{-1-\frac{1}{\alpha}} \rho_{\alpha}\left(\theta^{-\frac{1}{\alpha}}\right)$ is the probability density function defined on $(0, \infty)$.

For any $u \in E$, define operators $S_{\psi}^{\alpha}(t, s)$ and $T_{\psi}^{\alpha}(t, s)$ by

$$
S_{\psi}^{\alpha}(t, s) u=\int_{0}^{\infty} \phi_{\alpha}(\theta) T\left((\psi(t)-\psi(s))^{\alpha} \theta\right) u d \theta
$$

and

$$
T_{\psi}^{\alpha}(t, s) u=\alpha \int_{0}^{\infty} \theta \phi_{\alpha}(\theta) T\left((\psi(t)-\psi(s))^{\alpha} \theta\right) u d \theta
$$

for $0 \leq s \leq t \leq T$.

Lemma 3.2 The operators $S_{\psi}^{\alpha}$ and $T_{\psi}^{\alpha}$ have the following properties:

(i) For any fixed $t \geq s \geq 0, S_{\psi}^{\alpha}(t, s)$ and $T_{\psi}^{\alpha}(t, s)$ are bounded linear operators with

$$
\left\|S_{\psi}^{\alpha}(t, s)(u)\right\| \leq M\|u\| \quad \text { and } \quad\left\|T_{\psi}^{\alpha}(t, s)(u)\right\| \leq \frac{\alpha M}{\Gamma(1+\alpha)}\|u\|=\frac{M}{\Gamma(\alpha)}\|u\|
$$

for all $u \in E$.

(ii) The operators $S_{\psi}^{\alpha}(t, s)$ and $T_{\psi}^{\alpha}(t, s)$ are strongly continuous for all $t \geq s \geq 0$, that is, for every $u \in E$ and $0 \leq s \leq t_{1}<t_{2} \leq T$ we have

$$
\left\|S_{\psi}^{\alpha}\left(t_{2}, s\right) u-S_{\psi}^{\alpha}\left(t_{1}, s\right) u\right\| \rightarrow 0 \quad \text { and } \quad\left\|T_{\psi}^{\alpha}\left(t_{2}, s\right) u-T_{\psi}^{\alpha}\left(t_{1}, s\right) u\right\| \rightarrow 0
$$

as $t_{1} \rightarrow t_{2}$. 
(iii) If $T(t)$ is compact operator for every $t>0$, then $S_{\psi}^{\alpha}(t, s)$ and $T_{\psi}^{\alpha}(t, s)$ are compact for all $t, s>0$.

(iv) If $S_{\psi}^{\alpha}(t, s)$ and $T_{\psi}^{\alpha}(t, s)$ are compact strongly continuous semigroup of bounded linear operators for $t, s>0$, then $S_{\psi}^{\alpha}(t, s)$ and $T_{\psi}^{\alpha}(t, s)$ are continuous in the uniform operator topology.

Proof The proof follows the argument of [26].

Definition 3.3 A function $u \in C([0, T], E)$ is called a mild solution of (1) if it satisfies

$$
u(t)=S_{\psi}^{\alpha}(t, 0) u_{0}+\int_{0}^{t}(\psi(t)-\psi(s))^{\alpha-1} T_{\psi}^{\alpha}(t, s) f(s, u(s)) \psi^{\prime}(s) d s, \quad t \in[0, T]
$$

Before starting and proving the main results, we introduce the following hypotheses.

$\left(\mathrm{H}_{1}\right) T(t)$ is compact operator for every $t>0$.

$\left(\mathrm{H}_{2}\right)$ The function $f:[0, T] \times E \rightarrow E$ is Carathéodory function, that is:

$\left(C_{1}\right)$ For each $t \in[0, T]$ the function $f(t, \cdot): E \rightarrow E$ is continuous.

$\left(C_{2}\right)$ For each $u \in E$ the function $f(\cdot, u):[0, T] \rightarrow E$ is measurable.

$\left(\mathrm{H}_{3}\right)$ For any $r>0$, there exists a function $h_{r} \in L^{\infty}([0, T], E)$ such that

$$
\sup _{\|u\| \leq r}\|f(t, u)\| \leq h_{r}(t), \quad \text { a.e. } t \in[0, T]
$$

and there is a constant $L>0$ such that

$$
\limsup _{r \rightarrow \infty} \frac{\left\|h_{r}(t)\right\|_{L^{\infty}}}{r}=L
$$

$\left(\mathrm{H}_{4}\right)$ For any $r>0$, there exists $k(t) \in L^{\infty}([0, T], E)$ such that

$$
\left\|f\left(t, u_{1}(t)\right)-f\left(t, u_{2}(t)\right)\right\| \leq k(t)\left\|u_{1}-u_{2}\right\|
$$

for all $u_{1}, u_{2} \in \Omega_{r}$.

$\left(\mathrm{H}_{5}\right)$ There exist continuous functions $g_{1}, g_{2}$ on $[0, \infty)$ such that

$$
\|f(t, u)\| \leq g_{1}(t)+g_{2}(t)\|u\|
$$

for $t \geq 0$ and $u \in E$.

$\left(\mathrm{H}_{6}\right)$ For any $r>0$ and $T>0$, there exists a positive constant $K$ such that, for any equicontinuous and countable set $D \subset \Omega_{r}=\{u \in E \mid\|u\| \leq r\}$,

$$
\mu(f(t, D)) \leq K \mu(D), \quad t \in[0, T] .
$$

\section{Existence and uniqueness of mild solution under compact analytic semigroup}

In this section, we begin by proving a theorem concerning the existence and uniqueness of mild solution for the problem (1) under the condition of compact analytic semigroup. The discussions are based on fractional calculus and Schauder fixed point theorem. Our main results are as follows. 
Theorem 4.1 Assume that conditions $\left(\mathrm{H}_{1}\right)-\left(\mathrm{H}_{3}\right)$ hold. Then the problem (1) has at least mild solution provided that

$$
\frac{M L}{\Gamma(1+\alpha)}(\psi(T)-\psi(0))^{\alpha}<1 .
$$

Proof For any $r>0$, let $\Omega_{r}=\{u \in C([0, T], E):\|u\| \leq r\}$. Then $\Omega_{r}$ is bounded closed convex subset of $C([0, T], E)$. Define an operator $\mathcal{K}: \Omega_{r} \rightarrow C([0, T], E)$ by

$$
(\mathcal{K} u)(t):=S_{\psi}^{\alpha}(t, 0) u_{0}+\int_{0}^{t}(\psi(t)-\psi(s))^{\alpha-1} T_{\psi}^{\alpha}(t, s) f(s, u(s)) \psi^{\prime}(s) d s,
$$

for $t \in[0, T]$.

Step 1: We will prove that $\mathcal{K}: \Omega_{r} \rightarrow \Omega_{r}$, that is, there exists $r>0$ such that $\mathcal{K}\left(\Omega_{r}\right) \subset \Omega_{r}$, We assume that for each $r>0$, there exists $u_{r} \in \Omega_{r}$ and $t \in[0, T]$, such that $\|(\mathcal{K} u)(t)\|>r$. According to Lemma 3.2(i) and $\left(\mathrm{H}_{3}\right)$, we have

$$
\begin{aligned}
r & <\left\|\left(\mathcal{K} u_{r}\right)(t)\right\| \leq\left\|S_{\psi}^{\alpha}(t, 0) u_{0}\right\|+\int_{0}^{t}\left\|(\psi(t)-\psi(s))^{\alpha-1} T_{\psi}^{\alpha}(t, s) f\left(s, u_{r}(s)\right) \psi^{\prime}(s)\right\| d s \\
& \leq M\left\|u_{0}\right\|+\int_{0}^{t}(\psi(t)-\psi(s))^{\alpha-1}\left\|T_{\psi}^{\alpha}(t, s) f\left(s, u_{r}(s)\right)\right\| \psi^{\prime}(s) d s \\
& \leq M\left\|u_{0}\right\|+\frac{M}{\Gamma(\alpha)} \int_{0}^{t}(\psi(t)-\psi(s))^{\alpha-1}\left\|f\left(s, u_{r}(s)\right)\right\| \psi^{\prime}(s) d s \\
& \leq M\left\|u_{0}\right\|+\frac{M}{\Gamma(\alpha)} \int_{0}^{t}(\psi(t)-\psi(s))^{\alpha-1} h_{r}(s) \psi^{\prime}(s) d s \\
& \leq M\left\|u_{0}\right\|+\frac{M\left\|h_{r}(t)\right\|_{L^{\infty}}}{\Gamma(\alpha)} \int_{0}^{t}(\psi(t)-\psi(s))^{\alpha-1} \psi^{\prime}(s) d s \\
& =M\left\|u_{0}\right\|+\frac{M\left\|h_{r}(t)\right\|_{L^{\infty}}}{\Gamma(1+\alpha)}(\psi(t)-\psi(s))^{\alpha} \\
& \leq M\left\|u_{0}\right\|+\frac{M\left\|h_{r}(t)\right\|_{L^{\infty}}}{\Gamma(1+\alpha)}(\psi(T)-\psi(0))^{\alpha} .
\end{aligned}
$$

Dividing to both side by $r$ and taking the limit supremum as $r \rightarrow \infty$, we obtain

$$
\begin{aligned}
1 & \leq \limsup _{r \rightarrow \infty} \frac{M}{r}\left\|u_{0}\right\|+\limsup _{r \rightarrow \infty} \frac{M\left\|h_{r}(t)\right\|_{L^{\infty}}}{r \Gamma(1+\alpha)}(\psi(T)-\psi(0))^{\alpha} \\
& =\frac{M L}{\Gamma(1+\alpha)}(\psi(T)-\psi(0))^{\alpha}<1,
\end{aligned}
$$

which is contradiction. Therefore $\mathcal{K}: \Omega_{r} \rightarrow \Omega_{r}$.

Step 2: We will prove that $\mathcal{K}: \Omega_{r} \rightarrow \Omega_{r}$ is continuous. Let $\left\{u_{n}\right\} \subset \Omega_{r}$ with $u_{n} \rightarrow u \in \Omega_{r}$ as $n \rightarrow \infty$.

From the assumptions $\left(\mathrm{H}_{2}\right)$ and $\left(\mathrm{H}_{3}\right)$, we have, for each $t \in[0, T]$,

$$
f\left(t, u_{n}(t)\right) \rightarrow f(t, u(t)) \quad \text { as } n \rightarrow \infty
$$

and

$$
\left\|f\left(t, u_{n}(t)\right)-f(t, u(t))\right\| \leq 2 h_{r}(t) \quad \text { for all } n \in \mathbb{N} .
$$


By the Lebesgue dominated convergence theorem, for any $t \in[0, T]$ we have

$$
\begin{aligned}
& \left\|\left(\mathcal{K} u_{n}\right)(t)-(\mathcal{K} u)(t)\right\| \\
& \quad \leq \int_{0}^{t}(\psi(t)-\psi(s))^{\alpha-1}\left\|T_{\psi}^{\alpha}(t, s)\left[f\left(s, u_{n}(s)\right)-f(s, u(s))\right]\right\| \psi^{\prime}(s) d s \\
& \quad \leq \frac{M}{\Gamma(\alpha)} \int_{0}^{t}(\psi(t)-\psi(s))^{\alpha-1}\left\|f\left(s, u_{n}(s)\right)-f(s, u(s))\right\| \psi^{\prime}(s) d s \rightarrow 0
\end{aligned}
$$

as $n \rightarrow \infty$. Therefore, $\left\|\left(\mathcal{K} u_{n}\right)(t)-(\mathcal{K} u)(t)\right\|_{C} \rightarrow 0$ as $n \rightarrow \infty$. Hence $\mathcal{K}: \Omega_{r} \rightarrow \Omega_{r}$ is continuous.

Step 3: We will prove that $\mathcal{K}\left(\Omega_{r}\right)$ is equicontinuous. For any $u \in \Omega_{r}$ and $0 \leq t_{1}<t_{2} \leq T$, we have

$$
\begin{aligned}
\|(\mathcal{K} u) & \left(t_{2}\right)-(\mathcal{K} u)\left(t_{1}\right) \| \\
\leq & \left\|S_{\psi}^{\alpha}\left(t_{2}, 0\right) u_{0}-S_{\psi}^{\alpha}\left(t_{1}, 0\right) u_{0}\right\| \\
& +\| \int_{0}^{t_{2}}\left(\psi\left(t_{2}\right)-\psi(s)\right)^{\alpha-1} T_{\psi}^{\alpha}\left(t_{2}, s\right) f(s, u(s)) \psi^{\prime}(s) d s \\
& -\int_{0}^{t_{1}}\left(\psi\left(t_{1}\right)-\psi(s)\right)^{\alpha-1} T_{\psi}^{\alpha}\left(t_{1}, s\right) f(s, u(s)) \psi^{\prime}(s) d s \| \\
= & \left\|S_{\psi}^{\alpha}\left(t_{2}, 0\right) u_{0}-S_{\psi}^{\alpha}\left(t_{1}, 0\right) u_{0}\right\| \\
& +\| \int_{0}^{t_{1}}\left(\psi\left(t_{2}\right)-\psi(s)\right)^{\alpha-1} T_{\psi}^{\alpha}\left(t_{2}, s\right) f(s, u(s)) \psi^{\prime}(s) d s \\
& +\int_{t_{1}}^{t_{2}}\left(\psi\left(t_{2}\right)-\psi(s)\right)^{\alpha-1} T_{\psi}^{\alpha}\left(t_{2}, s\right) f(s, u(s)) \psi^{\prime}(s) d s \\
& +\int_{0}^{t_{1}}\left(\psi\left(t_{1}\right)-\psi(s)\right)^{\alpha-1} T_{\psi}^{\alpha}\left(t_{2}, s\right) f(s, u(s)) \psi^{\prime}(s) d s \\
& +\left\|\int_{0}^{t_{1}}\left(\psi\left(t_{1}\right)-\psi(s)\right)^{\alpha-1}\left[T_{\psi}^{\alpha}\left(t_{2}, s\right)-T_{\psi}^{\alpha}\left(t_{1}, s\right)\right] f(s, u(s)) \psi^{\prime}(s) d s\right\| \|{ }^{t_{1}}+I_{3}+I_{4} \cdot \\
& +\left\|\int_{t_{1}}^{t_{2}}\left(\psi\left(t_{2}\right)-\psi(s)\right)^{\alpha-1} T_{\psi}^{\alpha}\left(t_{2}, s\right) f(s, u(s)) \psi^{\prime}(s) d s\right\| \\
& -\int_{0}^{t_{1}}\left(\psi\left(t_{1}\right)-\psi(s)\right)^{\alpha-1} T_{\psi}^{\alpha}\left(t_{2}, s\right) f(s, u(s)) \psi^{\prime}(s) d s \\
& -\int_{0}^{t_{1}}\left(\psi\left(t_{1}\right)-\psi(s)\right)^{\alpha-1} T_{\psi}^{\alpha}\left(t_{1}, s\right) f(s, u(s)) \psi^{\prime}(s) d s \|
\end{aligned}
$$

By Lemma 3.2, it is clear that $I_{1} \rightarrow 0$ as $t_{1} \rightarrow t_{2}$ and we obtain

$$
I_{2} \leq \frac{M\left\|h_{r}\right\|_{L^{\infty}}}{\Gamma(\alpha+1)}\left(\psi\left(t_{2}\right)-\psi\left(t_{1}\right)\right)^{\alpha}
$$


and

$$
I_{3} \leq \frac{M\left\|h_{r}\right\|_{L^{\infty}}}{\Gamma(\alpha+1)}\left[\left(\psi\left(t_{2}\right)\right)^{\alpha}-\left(\psi\left(t_{1}\right)\right)^{\alpha}-\left(\psi\left(t_{2}\right)-\psi\left(t_{1}\right)\right)^{\alpha}\right]
$$

and hence $I_{2} \rightarrow 0$ and $I_{3} \rightarrow 0$ as $t_{2} \rightarrow t_{1}$. For $t_{1}=0$ and $0<t_{2} \leq T$, it easy to see that $I_{4}=0$. Then, for any $\varepsilon \in\left(0, t_{1}\right)$, we have

$$
\begin{aligned}
I_{4} \leq & \left\|\int_{0}^{t_{1}-\varepsilon}\left(\psi\left(t_{1}\right)-\psi(s)\right)^{\alpha-1}\left[T_{\psi}^{\alpha}\left(t_{2}, s\right)-T_{\psi}^{\alpha}\left(t_{1}, s\right)\right] f(s, u(s)) \psi^{\prime}(s) d s\right\| \\
& +\left\|\int_{t_{1}-\varepsilon}^{t_{1}}\left(\psi\left(t_{1}\right)-\psi(s)\right)^{\alpha-1}\left[T_{\psi}^{\alpha}\left(t_{2}, s\right)-T_{\psi}^{\alpha}\left(t_{1}, s\right)\right] f(s, u(s)) \psi^{\prime}(s) d s\right\| \\
\leq & \frac{\left\|h_{r}\right\|_{L^{\infty}}}{\alpha}\left[\left(\psi\left(t_{1}\right)-\psi(0)\right)^{\alpha}-\left(\psi\left(t_{1}\right)-\psi\left(t_{1}-\varepsilon\right)\right)^{\alpha}\right] \sup _{0 \leq s<t_{1}-\varepsilon}\left\|T_{\psi}^{\alpha}\left(t_{2}, s\right)-T_{\psi}^{\alpha}\left(t_{1}, s\right)\right\| \\
& +\frac{2 M\left\|h_{r}\right\|_{L^{\infty}}}{\Gamma(\alpha+1)}\left[\left(\psi\left(t_{1}\right)-\psi\left(t_{1}-\varepsilon\right)\right)^{\alpha}\right] .
\end{aligned}
$$

It follows that $I_{4} \rightarrow 0$ as $t_{2} \rightarrow t_{1}$ and $\varepsilon \rightarrow 0$ by Lemma 3.2(iv) and (iii). Therefore,

$$
\left\|(\mathcal{K} u)\left(t_{2}\right)-(\mathcal{K} u)\left(t_{1}\right)\right\| \rightarrow 0 \quad \text { independently of } u \in \Omega_{r} \text { as } t_{2} \rightarrow t_{1} .
$$

which means that $\mathcal{K}\left(\Omega_{r}\right)$ is equicontinuous.

Step 4: We will prove that, for any $t \in[0, T], K(t)=\left\{(\mathcal{K} u)(t): u \in \Omega_{r}\right\}$ is relatively compact in $E$.

Obviously, $K(0)$ is relatively compact in $E$. Let $0 \leq t \leq T$ be fixed. Then, for every $\varepsilon>0$ and $\delta>0$, let $u \in \Omega_{r}$ and define an operator $\mathcal{K}_{\varepsilon, \delta}$ on $\Omega_{r}$ by

$$
\begin{aligned}
\left(\mathcal{K}_{\varepsilon, \delta} u\right)(t) & \\
= & \int_{0}^{\infty} \phi_{\alpha}(\theta) T\left((\psi(t)-\psi(0))^{\alpha} \theta\right) u_{0} d \theta \\
& +\alpha \int_{0}^{t-\varepsilon} \int_{\delta}^{\infty} \theta \phi_{\alpha}(\theta)(\psi(t)-\psi(s))^{\alpha-1} T\left((\psi(t)-\psi(0))^{\alpha} \theta\right) f(s, u(s)) \psi^{\prime}(s) d \theta d s \\
= & \int_{0}^{\infty} \phi_{\alpha}(\theta) T\left((\psi(t)-\psi(0))^{\alpha} \theta\right) u_{0} d \theta \\
& +\alpha \int_{0}^{t-\varepsilon} \int_{\delta}^{\infty} \theta \phi_{\alpha}(\theta)(\psi(t)-\psi(s))^{\alpha-1} T\left((\psi(t)-\psi(0))^{\alpha} \theta+\varepsilon^{\alpha} \delta-\varepsilon^{\alpha} \delta\right) \\
& f(s, u(s)) \psi^{\prime}(s) d \theta d s \\
= & \int_{0}^{\infty} \phi_{\alpha}(\theta) T\left((\psi(t)-\psi(0))^{\alpha} \theta\right) u_{0} d \theta \\
& +\alpha \int_{0}^{t-\varepsilon} \int_{\delta}^{\infty} \theta \phi_{\alpha}(\theta)(\psi(t)-\psi(s))^{\alpha-1}\left[T\left(\varepsilon^{\alpha} \delta\right) T\left((\psi(t)-\psi(0))^{\alpha} \theta-\varepsilon^{\alpha} \delta\right)\right] \\
& f(s, u(s)) \psi^{\prime}(s) d \theta d s \\
= & \int_{0}^{\infty} \phi_{\alpha}(\theta) T\left((\psi(t)-\psi(0))^{\alpha} \theta\right) u_{0} d \theta
\end{aligned}
$$




$$
\begin{aligned}
+ & \alpha T\left(\varepsilon^{\alpha} \delta\right) \int_{0}^{t-\varepsilon} \int_{\delta}^{\infty} \theta \phi_{\alpha}(\theta)(\psi(t)-\psi(s))^{\alpha-1} T\left((\psi(t)-\psi(0))^{\alpha} \theta-\varepsilon^{\alpha} \delta\right) \\
& f(s, u(s)) \psi^{\prime}(s) d \theta d s .
\end{aligned}
$$

Then, by the compactness of $T\left(\varepsilon^{\alpha} \delta\right)$ for $\varepsilon^{\alpha} \delta>0$, we see that the set $K_{\varepsilon, \delta}(t)=\left\{\left(\mathcal{K}_{\varepsilon, \delta} u\right)(t)\right.$ : $\left.u \in \Omega_{r}\right\}$ is relatively compact in $E$ for all $\varepsilon>0$ and $\delta>0$. Furthermore, for any $u \in \Omega_{r}$, we have

$$
\begin{aligned}
& \left\|(\mathcal{K} u)(t)-\left(\mathcal{K}_{\varepsilon, \delta} u\right)(t)\right\| \\
& =\alpha \| \int_{0}^{t} \int_{0}^{\delta} \theta \phi_{\alpha}(\theta)(\psi(t)-\psi(s))^{\alpha-1} T\left((\psi(t)-\psi(0))^{\alpha} \theta\right) f(s, u(s)) \psi^{\prime}(s) d \theta d s \\
& +\int_{0}^{t} \int_{\delta}^{\infty} \theta \phi_{\alpha}(\theta)(\psi(t)-\psi(s))^{\alpha-1} T\left((\psi(t)-\psi(0))^{\alpha} \theta\right) f(s, u(s)) \psi^{\prime}(s) d \theta d s \\
& +\int_{0}^{t-\varepsilon} \int_{\delta}^{\infty} \theta \phi_{\alpha}(\theta)(\psi(t)-\psi(s))^{\alpha-1} T\left((\psi(t)-\psi(0))^{\alpha} \theta\right) f(s, u(s)) \psi^{\prime}(s) d \theta d s \| \\
& \leq \alpha\left\|\int_{0}^{t} \int_{0}^{\delta} \theta \phi_{\alpha}(\theta)(\psi(t)-\psi(s))^{\alpha-1} T\left((\psi(t)-\psi(0))^{\alpha} \theta\right) f(s, u(s)) \psi^{\prime}(s) d \theta d s\right\| \\
& +\alpha \| \int_{t-\varepsilon}^{t} \int_{\delta}^{\infty} \theta \phi_{\alpha}(\theta)(\psi(t)-\psi(s))^{\alpha-1} T\left((\psi(t)-\psi(0))^{\alpha} \theta\right) \\
& f(s, u(s)) \psi^{\prime}(s) d \theta d s \| \theta d s \\
& \leq \alpha M\left\|h_{r}\right\|_{L^{\infty}}\left(\int_{0}^{t}(\psi(t)-\psi(s))^{\alpha-1} \psi^{\prime}(s) d s\right)\left(\int_{0}^{\delta} \theta \phi_{\alpha}(\theta) d \theta\right) \\
& +\alpha M\left\|h_{r}\right\|_{L^{\infty}}\left(\int_{t-\varepsilon}^{t}(\psi(t)-\psi(s))^{\alpha-1} \psi^{\prime}(s) d s\right)\left(\int_{\delta}^{\infty} \theta \phi_{\alpha}(\theta) d \theta\right) \\
& =M\left\|h_{r}\right\|_{L^{\infty}}(\psi(t)-\psi(0))^{\alpha}\left(\int_{0}^{\delta} \theta \phi_{\alpha}(\theta) d \theta\right) \\
& +M\left\|h_{r}\right\|_{L^{\infty}}(\psi(t)-\psi(t-\varepsilon))^{\alpha}\left(\int_{\delta}^{\infty} \theta \phi_{\alpha}(\theta) d \theta\right) \\
& \leq M\left\|h_{r}\right\|_{L^{\infty}}(\psi(t)-\psi(0))^{\alpha}\left(\int_{0}^{\delta} \theta \phi_{\alpha}(\theta) d \theta\right) \\
& +M\left\|h_{r}\right\|_{L^{\infty}}(\psi(t)-\psi(t-\varepsilon))^{\alpha}\left(\int_{0}^{\infty} \theta \phi_{\alpha}(\theta) d \theta\right) \\
& =M\left\|h_{r}\right\|_{L^{\infty}}(\psi(t)-\psi(0))^{\alpha}\left(\int_{0}^{\delta} \theta \phi_{\alpha}(\theta) d \theta\right) \\
& +\frac{M\left\|h_{r}\right\|_{L^{\infty}}}{\Gamma(\alpha+1)}(\psi(t)-\psi(t-\varepsilon))^{\alpha} \\
& \leq M\left\|h_{r}\right\|_{L^{\infty}}(\psi(T)-\psi(0))^{\alpha}\left(\int_{0}^{\delta} \theta \phi_{\alpha}(\theta) d \theta\right)+\frac{M\left\|h_{r}\right\|_{L^{\infty}}}{\Gamma(\alpha+1)}(\psi(t)-\psi(t-\varepsilon))^{\alpha} \\
& \rightarrow 0 \quad \text { as } \varepsilon, \delta \rightarrow 0^{+} \text {. }
\end{aligned}
$$

Therefore, there are relatively compact sets arbitrarily close to the set $K(t)$ for $t>0$. Hence, $K(t)$ is relatively compact in $E$. 
Therefore, by the Arzelá-Ascoli theorem $\mathcal{K}\left(\Omega_{r}\right)$ is relatively compact in $C([0, T], E)$. Thus, the continuity of $\mathcal{K}$ and relatively compact of $\mathcal{K}\left(\Omega_{r}\right)$ imply that $\mathcal{K}$ is a completely continuous. By the Schauder fixed point theorem, we see that $\mathcal{K}$ has a fixed point $u^{*}$ in $\Omega_{r}$, which is a mild solution of (1). The proof is complete.

Remark 4.2 From Theorem 4.1, we notice that if $\psi$ is bijection function then the problem (1) has at least mild solution provided that

$$
T<\psi^{-1}\left[\left(\frac{\Gamma(1+\alpha)}{M L}\right)^{\frac{1}{\alpha}}+\psi(0)\right]
$$

Theorem 4.3 Assume $\left(\mathrm{H}_{4}\right)$ holds. Then the problem (1) has a unique mild solution.

Proof Let $u_{1}$ and $u_{2}$ be the solutions of the problem (1) in $\Omega_{r}$. Then, for each $i \in\{1,2\}$, the solution $u_{i}$ satisfies

$$
\left(\mathcal{K} u_{i}\right)(t):=S_{\psi}^{\alpha}(t, 0) u_{0}+\int_{0}^{t}(\psi(t)-\psi(s))^{\alpha-1} T_{\psi}^{\alpha}(t, s) f\left(s, u_{i}(s)\right) \psi^{\prime}(s) d s .
$$

Then, for any $t \in[0, T]$, we have

$$
\begin{aligned}
\left\|u_{1}(t)-u_{2}(t)\right\| & =\left\|\left(\mathcal{K} u_{1}\right)(t)-\left(\mathcal{K} u_{2}\right)(t)\right\| \\
& \leq \int_{0}^{t}(\psi(t)-\psi(s))^{\alpha-1}\left\|T_{\psi}^{\alpha}(t, s) f\left(s, u_{1}(s)\right)-f\left(s, u_{2}(s)\right)\right\| \psi^{\prime}(s) d s \\
& \leq \frac{M}{\Gamma(\alpha)} \int_{0}^{t}(\psi(t)-\psi(s))^{\alpha-1}\left\|\left[f\left(s, u_{1}(s)\right)-f\left(s, u_{2}(s)\right)\right]\right\| \psi^{\prime}(s) d s \\
& \leq \frac{M}{\Gamma(\alpha)} \int_{0}^{t}(\psi(t)-\psi(s))^{\alpha-1} k(s)\left\|u_{1}(s)-u_{2}(s)\right\| \psi^{\prime}(s) d s \\
& \leq \frac{M k^{*}}{\Gamma(\alpha)} \int_{0}^{t}(\psi(t)-\psi(s))^{\alpha-1}\left\|u_{1}(s)-u_{2}(s)\right\| \psi^{\prime}(s) d s,
\end{aligned}
$$

where $k^{*}=\sup _{0 \leq t \leq T}|k(t)|$. By using the Gronwall inequality (Lemma 2.11), we obtain

$$
\left\|u_{1}(t)-u_{2}(t)\right\|=0 \quad \text { for all } t \in[0, T]
$$

which implies that $u_{1} \equiv u_{2}$. Therefore, the problem (1) has a unique mild solution $u^{*} \in$ $\Omega_{r}$.

Theorem 4.4 Suppose that conditions $\left(\mathrm{H}_{1}\right)-\left(\mathrm{H}_{3}\right)$ hold. Then, for any $u_{0} \in E$, the problem (1) has a mild solution $u$ on a maximal interval of existence $\left[0, T_{\max }\right)$. If $T_{\max }<\infty$, then $\lim _{t \rightarrow T_{\max }^{-}}\|u(t)\|=\infty$.

Proof We notice that a mild solution $u$ of the problem (1) defined on $[0, T]$ can be extended to a larger interval $[0, T+\delta]$ with $\delta>0$, by defining $v(t)=u(t+T)$, where $v(t)$ is a mild solution of

$$
\left\{\begin{array}{l}
{ }_{0}^{\mathrm{C}} D_{\psi}^{\alpha} v(t)=A v(t)+f(t, v(t)), \quad t \in(T, T+\delta], \\
v(0)=u(T) .
\end{array}\right.
$$


Therefore, repeating the procedure and using the methods of steps in Theorem 4.1, we can prove that there exists a maximal interval $\left[0, T_{\max }\right)$ such that the mild solution $u$ of the problem (1). We want to prove that if $T_{\max }<\infty$ then $\lim _{t \rightarrow T_{\max }^{-}}\|u(t)\|=\infty$.

First, we will prove that $\limsup _{t \rightarrow T_{\max }^{-}}\|u(t)\|=\infty$. Assume by contradiction that $\lim \sup _{t \rightarrow T_{\max }^{-}}\|u(t)\|<\infty$. Then there exists $K>0$ such that $\|u(t)\| \leq K$ for $0 \leq t<T_{\max }$. For $0<t<t^{\prime}<T_{\max }$, we have

$$
\begin{aligned}
\| u\left(t^{\prime}\right) & -u(t) \| \\
\leq & \left\|S_{\psi}^{\alpha}\left(t^{\prime}, 0\right) u_{0}-S_{\psi}^{\alpha}(t, 0) u_{0}\right\| \\
& +\left\|\int_{t}^{t^{\prime}}\left(\psi\left(t^{\prime}\right)-\psi(s)\right)^{\alpha-1} T_{\psi}^{\alpha}\left(t^{\prime}, s\right) f(s, u(s)) \psi^{\prime}(s) d s\right\| \\
& +\left\|\int_{0}^{t}\left[\left(\psi\left(t^{\prime}\right)-\psi(s)\right)^{\alpha-1}-(\psi(t)-\psi(s))^{\alpha-1}\right] T_{\psi}^{\alpha}\left(t^{\prime}, s\right) f(s, u(s)) \psi^{\prime}(s) d s\right\| \\
& +\left\|\int_{0}^{t}(\psi(t)-\psi(s))^{\alpha-1}\left[T_{\psi}^{\alpha}\left(t^{\prime}, s\right)-T_{\psi}^{\alpha}(t, s)\right] f(s, u(s)) \psi^{\prime}(s) d s\right\| \\
= & : I_{1}+I_{2}+I_{3}+I_{4} .
\end{aligned}
$$

Similar to Step 3 of Theorem 4.1, we can prove that $\left\|u\left(t^{\prime}\right)-u(t)\right\| \rightarrow 0$ as $t^{\prime}, t \rightarrow T_{\max }$ Therefore, by the Cauchy criteria we see that $\lim _{t \rightarrow T_{\max }^{-}} u(t)=u_{1}$ exists. By the first part of the proof, there exists a $\delta>0$ such that the solution can be extended to $\left[0, T_{\max }+\delta\right.$ ) and we know that to the fractional evolution equation

$$
\left\{\begin{array}{l}
{ }_{0}^{C} D_{\psi}^{\alpha} u(t)=A u(t)+f(t, u(t)), \quad 0 \leq t<\delta, \\
u\left(T_{\max }\right)=u_{1},
\end{array}\right.
$$

there exists a mild solution on $\left[T_{\max }, T_{\max }+\delta\right.$ ). This means that the mild solution of the problem (1) can be extended to $\left[0, T_{\max }+\delta\right.$ ), which contradicts with the maximal interval $\left[0, T_{\max }\right)$. Hence, $\limsup _{t \rightarrow T_{\max }^{-}}\|u(t)\|=\infty$.

Now, we will prove that if $T_{\max }<\infty$, then $\lim _{t \rightarrow T_{\max }^{-}}\|u(t)\|=\infty$. If this is not true, then there exist a constant $K>0$ and a sequence $t_{n} \rightarrow T_{\max }$ such that $\left\|u\left(t_{n}\right)\right\| \leq K$ for all $n$. Since $t \rightarrow\|u(t)\|$ is continuous and $\lim \sup _{t \rightarrow T_{\max }^{-}}\|u(t)\|=\infty$, we can find a sequence $a_{n}$ such that $a_{n} \rightarrow 0$ as $n \rightarrow \infty,\|u(t)\| \leq M(K+1)$ for $t_{n} \leq t \leq t_{n}+a_{n}$ and $\left\|u\left(t_{n}+a_{n}\right)\right\|=M(K+1)$ for all $n$ sufficiently large. But we have

$$
\begin{aligned}
M(K+1)= & \left\|u\left(t_{n}+a_{n}\right)\right\| \\
\leq & \left\|S_{\psi}^{\alpha}\left(a_{n}, 0\right) u\left(t_{n}\right)\right\| \\
& +\int_{t_{n}}^{t_{n}+a_{n}}\left\|\left(\psi\left(t_{n}+a_{n}\right)-\psi(s)\right)^{\alpha-1} T_{\psi}^{\alpha}\left(t_{n}+a_{n}, s\right) f(s, u(s)) \psi^{\prime}(s)\right\| d s \\
\leq & M K+\frac{M}{\Gamma(\alpha)}\left\|h_{r}(t)\right\|_{L^{\infty}} \int_{t_{n}}^{t_{n}+a_{n}}\left(\psi\left(t_{n}+a_{n}\right)-\psi(s)\right)^{\alpha-1} \psi^{\prime}(s) d s \\
\leq & M K+\frac{M}{\Gamma(\alpha+1)}\left\|h_{r}(t)\right\|_{L^{\infty}}\left(\psi\left(t_{n}+a_{n}\right)-\psi\left(t_{n}\right)\right)^{\alpha},
\end{aligned}
$$


which implies that $M(K+1) \leq M K$ as $a_{n} \rightarrow 0$, a contradiction. Therefore, we find that if $T_{\max }<\infty$, then $\lim _{t \rightarrow T_{\max }^{-}}\|u(t)\|=\infty$.

Next, we discuss the existence of a global mild solution for the problem (1). To this end, we need replace the assumption $\left(\mathrm{H}_{3}\right)$ by $\left(\mathrm{H}_{5}\right)$.

Theorem 4.5 Assume that conditions $\left(\mathrm{H}_{1}\right)-\left(\mathrm{H}_{2}\right)$ and $\left(\mathrm{H}_{5}\right)$ hold, then for every $u_{0} \in E$ the problem (1) has a global mild solution $u \in C([0, \infty), E)$.

Proof It is clearly that $\left(\mathrm{H}_{5}\right)$ implies $\left(\mathrm{H}_{3}\right)$. Therefore, by Theorem 4.4 we know that the problem (1) has a mild solution $u$ on a maximal interval of existence $\left[0, T_{\max }\right)$. By the proof process of Theorem 4.4, we can see that the problem (1) has a global mild solution if $u(t)$ is bounded for every $t$ in the interval of existence of $u$. If suffices to show that $u(t)$ is bounded for every $t \in\left[0, T_{\max }\right)$ with $T_{\max }<\infty$.

Then for any $0 \leq t \leq T_{\max }$ we have

$$
\begin{aligned}
\|u(t)\| \leq & \left\|S_{\psi}^{\alpha}(t, 0) u_{0}\right\|+\int_{0}^{t}(\psi(t)-\psi(s))^{\alpha-1}\left\|T_{\psi}^{\alpha}(t, s) f(s, u(s))\right\| \psi^{\prime}(s) d s \\
\leq & M\left\|u_{0}\right\|+\frac{\alpha M}{\Gamma(\alpha+1)} \int_{0}^{t}(\psi(t)-\psi(s))^{\alpha-1}\|f(s, u(s))\| \psi^{\prime}(s) d s \\
\leq & M\left\|u_{0}\right\|+\frac{\alpha M}{\Gamma(\alpha+1)} \int_{0}^{t}(\psi(t)-\psi(s))^{\alpha-1}\left(g_{1}(t)+g_{2}(t)\|u\|\right) \psi^{\prime}(s) d s \\
\leq & M\left\|u_{0}\right\|+\frac{M}{\Gamma(\alpha+1)} C_{1}(\psi(t)-\psi(0))^{\alpha} \\
& +\frac{M}{\Gamma(\alpha)} C_{2} \int_{0}^{t}(\psi(t)-\psi(s))^{\alpha-1}\|u(s)\| \psi^{\prime}(s) d s \\
\leq & M\left\|u_{0}\right\|+\frac{M}{\Gamma(\alpha+1)} C_{1}\left(\psi\left(T_{\max }\right)-\psi(0)\right)^{\alpha} \\
& +\frac{M}{\Gamma(\alpha)} C_{2} \int_{0}^{t}(\psi(t)-\psi(s))^{\alpha-1}\|u(s)\| \psi^{\prime}(s) d s \\
:= & K_{1}+K_{2} \int_{0}^{t}(\psi(t)-\psi(s))^{\alpha-1}\|u(s)\| \psi^{\prime}(s) d s,
\end{aligned}
$$

where

$$
C_{1}=\sup _{0 \leq t \leq T_{\max }} g_{1}(t), \quad C_{2}=\sup _{0 \leq t \leq T_{\max }} g_{2}(t)
$$

and

$$
K_{1}=M\left\|u_{0}\right\|+\frac{M}{\Gamma(\alpha+1)} C_{1}\left(\psi\left(T_{\max }\right)-\psi(0)\right)^{\alpha}, \quad K_{2}=\frac{M}{\Gamma(\alpha)} C_{2} .
$$

By Corollary 2.12, we obtain

$$
\begin{aligned}
\|u(t)\| & \leq K_{1} E_{\alpha}\left(K_{2} \Gamma(\alpha)[\psi(t)-\psi(0)]^{\alpha}\right) \\
& \leq K_{1} E_{\alpha}\left(K_{2} \Gamma(\alpha)\left[\psi\left(T_{\max }\right)-\psi(0)\right]^{\alpha}\right),
\end{aligned}
$$

which means that $u(t)$ is bounded for every $t \in\left[0, T_{\max }\right)$. 


\section{Existence and uniqueness of mild solution under noncompact analytic semigroup}

In this section, we will prove the existence of mild solution for the problem (1) under the condition of a noncompact analytic semigroup.

Theorem 5.1 Assume that conditions $\left(\mathrm{H}_{2}\right)-\left(\mathrm{H}_{3}\right)$ and $\left(\mathrm{H}_{6}\right)$ hold. Then the problem (1) has at least one mild solution provided that

$$
\frac{M L}{\Gamma(1+\alpha)}(\psi(T)-\psi(0))^{\alpha}<1 \quad \text { and } \quad \frac{4 M K}{\Gamma(1+\alpha)}(\psi(T)-\psi(0))^{\alpha}<1 .
$$

Proof For any $r>0$, let $\Omega_{r}=\{u \in C([0, T], E):\|u\| \leq r\}$.

Then, $\Omega_{r}$ is bounded closed convex subset of $C([0, T], E)$. Define an operator $\mathcal{K}: \Omega_{r} \rightarrow$ $C([0, T], E)$ by

$$
(\mathcal{K} u)(t):=S_{\psi}^{\alpha}(t, 0) u_{0}+\int_{0}^{t}(\psi(t)-\psi(s))^{\alpha-1} T_{\psi}^{\alpha}(t, s) f(s, u(s)) \psi^{\prime}(s) d s, \quad t \in[0, T] .
$$

Using the same argument in Theorem 4.1, we obtain $\mathcal{K}: \Omega_{r} \rightarrow \Omega_{r}$ is continuous and $\mathcal{K}\left(\Omega_{r}\right)$ is equicontinuous. Then it is sufficient to prove that $\mathcal{K}: \Omega_{r} \rightarrow \Omega_{r}$ is condensing.

Let $D=\overline{\mathrm{Co}} \mathcal{K}\left(\Omega_{r}\right)$, where $\overline{\mathrm{Co}}$ is the closure of convex hull. Then, by Lemma 2.21 we obtain $\overline{C o} \mathcal{K}\left(\Omega_{r}\right) \subset \Omega_{r}$ is bounded and equicontinuous. Now, we will prove that $\mathcal{K}: D \rightarrow D$ is a condensing operator. For any $D \subset \overline{\mathrm{Co}} \mathcal{K}\left(\Omega_{r}\right)$, by Lemma 2.17 , we see that there exists a countable set $D_{0}=\left\{u_{n}\right\} \subset D$ such that

$$
\mu(\mathcal{K}(D)) \leq 2 \mu\left(\mathcal{K}\left(D_{0}\right)\right)
$$

By the equicontinuity of $D$, we know that $D_{0} \subset D$ is also equicontinuous. Therefore, by Lemma 2.20, we have

$$
\begin{aligned}
\mu\left(\mathcal{K}\left(D_{0}\right)(t)\right)= & \mu\left(\left\{S_{\psi}^{\alpha}(t, 0) u_{0}+\int_{0}^{t}(\psi(t)-\psi(s))^{\alpha-1} T_{\psi}^{\alpha}(t, s) f\left(s, u_{n}(s)\right) \psi^{\prime}(s) d s\right\}\right) \\
\leq & \mu\left(\left\{S_{\psi}^{\alpha}(t, 0) u_{0}\right\}\right) \\
& +\mu\left(\left\{\int_{0}^{t}(\psi(t)-\psi(s))^{\alpha-1} T_{\psi}^{\alpha}(t, s) f\left(s, u_{n}(s)\right) \psi^{\prime}(s) d s\right\}\right) \\
\leq & 2 \int_{0}^{t} \mu\left(\left\{(\psi(t)-\psi(s))^{\alpha-1} T_{\psi}^{\alpha}(t, s) f\left(s, u_{n}(s)\right) \psi^{\prime}(s)\right\}\right) d s \\
\leq & \frac{2 M}{\Gamma(\alpha)} \int_{0}^{t}(\psi(t)-\psi(s))^{\alpha-1} \mu\left(\left\{f\left(s, u_{n}(s)\right)\right\}\right) \psi^{\prime}(s) d s \\
\leq & \frac{2 M}{\Gamma(\alpha)} \int_{0}^{t}(\psi(t)-\psi(s))^{\alpha-1} K \mu\left(D_{0}(s)\right) \psi^{\prime}(s) d s \\
= & \frac{2 M K}{\Gamma(\alpha)} \int_{0}^{t}(\psi(t)-\psi(s))^{\alpha-1} \psi^{\prime}(s) d s \cdot \mu(D) \\
= & \frac{2 M K}{\Gamma(\alpha+1)}(\psi(t)-\psi(0))^{\alpha} \mu(D) \\
\leq & \frac{2 M K}{\Gamma(\alpha+1)}(\psi(T)-\psi(0))^{\alpha} \mu(D) .
\end{aligned}
$$


Since $\mathcal{K}\left(D_{0}\right) \subset D$ is bounded and equicontinuous, we obtain

$$
\mu\left(\mathcal{K}\left(D_{0}\right)\right)=\max _{t \in J} \mu\left(D_{0}(t)\right)
$$

by Lemma (2.18). It follows that

$$
\begin{aligned}
\mu(\mathcal{K}(D)) & \leq 2 \mu\left(\mathcal{K}\left(D_{0}\right)\right) \\
& \leq \frac{4 M K}{\Gamma(\alpha+1)}(\psi(T)-\psi(0))^{\alpha} \cdot \mu(D) \\
& <\mu(D) .
\end{aligned}
$$

Thus, $\mathcal{K}: D \rightarrow D$ is a condensing operator. Therefore, by Lemma $2.22, \mathcal{K}$ has at least one fixed point $u^{*}$ in $\Omega_{r}$, which is a mild solution of (1). The proof is complete.

Remark 5.2 From Theorem 5.1, we notice that if $\psi$ is bijection function then the problem (1) has at least one mild solution provided that

$$
T<\min \left\{\psi^{-1}\left[\left(\frac{\Gamma(1+\alpha)}{M L}\right)^{\frac{1}{\alpha}}+\psi(0)\right], \psi^{-1}\left[\left(\frac{\Gamma(1+\alpha)}{4 M K}\right)^{\frac{1}{\alpha}}+\psi(0)\right]\right\} .
$$

Theorem 5.3 Assume that conditions $\left(\mathrm{H}_{2}\right)-\left(\mathrm{H}_{3}\right)$ and $\left(\mathrm{H}_{6}\right)$ hold. Then, for any $u_{0} \in E$, the problem (1) has a mild solution $u$ on a maximal interval of existence $\left[0, T_{\max }\right)$. If $T_{\max }<\infty$, then $\lim _{t \rightarrow T_{\max }^{-}}\|u(t)\|=\infty$.

Proof The proof uses the same argument as in Theorem 4.4.

Theorem 5.4 Assume that conditions $\left(\mathrm{H}_{2}\right)$ and $\left(\mathrm{H}_{5}\right)$ hold, then for every $u_{0} \in E$ the problem (1) has a global mild solution $u \in C([0, \infty), E)$.

Proof The proof uses the same argument as in Theorem 4.5.

\section{Mittag-Leffler-Ulam-Hyers stability}

For $f \in([0, T] \times E, E), \varphi \in C\left([0, T], \mathbb{R}^{+}\right)$and $\varepsilon>0$ we consider the equation

$$
{ }_{0}^{\mathrm{C}} D_{\psi}^{\alpha} u(t)=\mathcal{A} u(t)+f(t, u(t)), \quad t \in[0, T]
$$

and the inequalities

$$
\begin{aligned}
& \left|{ }_{0}^{C} D_{\psi}^{\alpha} u(t)-\mathcal{A} u(t)-f(t, u(t))\right| \leq \varepsilon, \quad t \in[0, T], \\
& \left|{ }_{0}^{C} D_{\psi}^{\alpha} u(t)-\mathcal{A} u(t)-f(t, u(t))\right| \leq \varphi(t), \quad t \in[0, T], \\
& \left|{ }_{0}^{C} D_{\psi}^{\alpha} u(t)-\mathcal{A} u(t)-f(t, u(t))\right| \leq \varepsilon \varphi(t), \quad t \in[0, T] .
\end{aligned}
$$

Definition 6.1 Equation (14) is Mittag-Leffler-Ulam-Hyers stable, with respect to $E_{\alpha}$, if there exists a real number $C>0$ such that for each $\varepsilon>0$ and for each solution $v \in$ $C^{1}([0, T], E)$ of inequality (15) there exists a mild solution $u \in C([0, T], E)$ of Eq. (14) with

$$
|v(t)-u(t)| \leq C \varepsilon E_{\alpha}(t), \quad t \in[0, T]
$$


Definition 6.2 Equation (14) is generalized Mittag-Leffler-Ulam-Hyers stable, with respect to $E_{\alpha}$, if there exists a function $\theta \in C\left(\mathbb{R}^{+}, \mathbb{R}^{+}\right), \theta(0)=0$, such that for each $\varepsilon>0$ and for each solution $v \in C^{1}([0, T], E)$ of inequality (15) there exists a mild solution $u \in C([0, T], E)$ of Eq. (14) with

$$
|v(t)-u(t)| \leq C \theta(\varepsilon) E_{\alpha}(t), \quad t \in[0, T]
$$

Definition 6.3 Equation (14) is Mittag-Leffler-Ulam-Hyers-Rassias stable, with respect to $\varphi E_{\alpha}$, if there exists a real number $C_{\varphi}>0$ such that for each $\varepsilon>0$ and for each solution $v \in C^{1}([0, T], E)$ of inequality (17) there exists a mild solution $u \in C([0, T], E)$ of Eq. (14) with

$$
|v(t)-u(t)| \leq C_{\varphi} \varepsilon \varphi(t) E_{\alpha}(t), \quad t \in[0, T] .
$$

Definition 6.4 Equation (14) is generalized Mittag-Leffler-Ulam-Hyers-Rassias stable, with respect to $\varphi E_{\alpha}$, if there exists a real number $C_{\varphi}>0$ such that for each solution $v \in$ $C^{1}([0, T], E)$ of inequality (16) there exists a mild solution $u \in C([0, T], E)$ of Eq. (14) with

$$
|v(t)-u(t)| \leq C_{\varphi} \varphi(t) E_{\alpha}(t), \quad t \in[0, T] .
$$

Remark 6.5 It is clear that Definition 6.1 implies 6.2 and 6.3 implies 6.4.

Remark 6.6 A function $u \in C^{1}([0, T], E)$ is a solution of the inequality (15) if and only if there exists a function $g \in C^{1}([0, T], E)$ (which depend on $u$ ) such that

(i) $|g(t)| \leq \varepsilon$ for $t \in[0, T]$,

(ii) ${ }_{0}^{C} D_{\psi}^{\alpha} u(t)=\mathcal{A} u(t)+f(t, u(t))+g(t), t \in[0, T]$.

Remark 6.7 If $v \in C^{1}([0, T], E)$ is a solution of inequality (15), $v$ is a solution of the following integral inequality:

$$
\begin{aligned}
& \left|v(t)-S_{\psi}^{\alpha}(t, 0) v(0)-\int_{0}^{t}(\psi(t)-\psi(s))^{\alpha-1} T_{\psi}^{\alpha}(t, s) f(s, v(s)) \psi^{\prime}(s) d s\right| \\
& \leq \varepsilon \int_{0}^{t}(\psi(t)-\psi(s))^{\alpha-1}\left\|T_{\psi}^{\alpha}(t, s)\right\| \psi^{\prime}(s) d s .
\end{aligned}
$$

Theorem 6.8 Assume that $f \in C([0, T] \times E, E)$ and there exists $L_{f}>0$ such that

$$
\left|f\left(t, u_{1}\right)-f\left(t, u_{2}\right)\right| \leq L_{f}\left|u_{1}-u_{2}\right|
$$

for all $t \in[0, T]$ and $u_{1}, u_{2} \in E$. Then, Eq. (14) is Mittag-Leffler-Ulam-Hyers stable.

Proof Let $v \in C^{1}([0, T], E)$ be a solution of inequality (15). Let us denote by $u \in C([0, T], E)$ the unique mild solution of the Cauchy problem

$$
\left\{\begin{array}{l}
{ }_{0}^{C} D_{\psi}^{\alpha} u(t)=\mathcal{A} u(t)+f(t, u(t)), \quad t \in(0, T] \\
u(0)=v(0) .
\end{array}\right.
$$


We have

$$
u(t)=S_{\psi}^{\alpha}(t, 0) v(0)+\int_{0}^{t}(\psi(t)-\psi(s))^{\alpha-1} T_{\psi}^{\alpha}(t, s) f(s, u(s)) \psi^{\prime}(s) d s, \quad t \in[0, T] .
$$

Then we get

$$
\begin{aligned}
\mid v(t) & -S_{\psi}^{\alpha}(t, 0) v(0)-\int_{0}^{t}(\psi(t)-\psi(s))^{\alpha-1} T_{\psi}^{\alpha}(t, s) f(s, v(s)) \psi^{\prime}(s) d s \mid \\
& \leq \varepsilon \int_{0}^{t}(\psi(t)-\psi(s))^{\alpha-1}\left\|T_{\psi}^{\alpha}(t, s)\right\| \psi^{\prime}(s) d s \\
& \leq \frac{\alpha M \varepsilon}{\Gamma(1+\alpha)} \int_{0}^{t}(\psi(t)-\psi(s))^{\alpha-1} \psi^{\prime}(s) d s \\
& =\frac{M}{\Gamma(1+\alpha)}(\psi(t)-\psi(0))^{\alpha} \varepsilon \\
& \leq \frac{M}{\Gamma(1+\alpha)}(\psi(T)-\psi(0))^{\alpha} \varepsilon .
\end{aligned}
$$

It follows that

$$
\begin{aligned}
\mid v(t) & -u(t) \mid \\
\leq & \left|v(t)-S_{\psi}^{\alpha}(t, 0) v(0)-\int_{0}^{t}(\psi(t)-\psi(s))^{\alpha-1} T_{\psi}^{\alpha}(t, s) f(s, u(s)) \psi^{\prime}(s) d s\right| \\
\leq & \left|v(t)-S_{\psi}^{\alpha}(t, 0) v(0)-\int_{0}^{t}(\psi(t)-\psi(s))^{\alpha-1} T_{\psi}^{\alpha}(t, s) f(s, v(s)) \psi^{\prime}(s) d s\right| \\
& +\mid \int_{0}^{t}(\psi(t)-\psi(s))^{\alpha-1} T_{\psi}^{\alpha}(t, s) f(s, v(s)) \psi^{\prime}(s) d s \\
& -\int_{0}^{t}(\psi(t)-\psi(s))^{\alpha-1} T_{\psi}^{\alpha}(t, s) f(s, u(s)) \psi^{\prime}(s) d s \mid \\
\leq & \left|v(t)-S_{\psi}^{\alpha}(t, 0) v(0)-\int_{0}^{t}(\psi(t)-\psi(s))^{\alpha-1} T_{\psi}^{\alpha}(t, s) f(s, v(s)) \psi^{\prime}(s) d s\right| \\
& +\int_{0}^{t}(\psi(t)-\psi(s))^{\alpha-1}\left\|T_{\psi}^{\alpha}(t, s)\right\||f(s, v(s))-f(s, u(s))| \psi^{\prime}(s) d s \\
\leq & \frac{M}{\Gamma(1+\alpha)}(\psi(T)-\psi(0))^{\alpha} \varepsilon \\
& +\frac{\alpha M L_{f}}{\Gamma(1+\alpha)} \int_{0}^{t}(\psi(t)-\psi(s))^{\alpha-1}|v(s)-u(s)| \psi^{\prime}(s) d s .
\end{aligned}
$$

By Corollary 2.12, we obtain

$$
|v(t)-u(t)| \leq \frac{M}{\Gamma(1+\alpha)}(\psi(T)-\psi(0))^{\alpha} E_{\alpha}\left(M L_{f}[\psi(t)-\psi(0)]^{\alpha}\right) \varepsilon .
$$

The proof is complete.

Theorem 6.9 Assume that the following conditions hold:

(i) $f \in C([0, \infty) \times E, E)$. 
(ii) $k(t)$ is a nonnegative, nondecreasing continuous function defined on $t \in[0, \infty)$ and

$$
\left|f\left(t, u_{1}\right)-f\left(t, u_{2}\right)\right| \leq k(t)\left|u_{1}-u_{2}\right|
$$

for all $t \in[0, \infty)$ and $u_{1}, u_{2} \in E$.

(iii) The function $\varphi \in C\left([0, \infty), \mathbb{R}^{+}\right)$is increasing and there exists $\lambda>0$ such that

$$
\int_{0}^{t}(\psi(t)-\psi(s))^{\alpha-1}\left\|T_{\phi}^{\alpha}(t, s)\right\| \varphi(s) \psi^{\prime}(s) d s \leq \lambda \varphi(t)
$$

for all $t \in[0, \infty)$. Then, Eq. (14) is generalized Mittag-Leffler-Ulam-Hyers-Rassias stable with respect to $\varphi E_{\alpha}$.

Proof Let $v \in C^{1}([0, T], \infty)$ be a solution of inequality (16). Then we get

$$
\begin{aligned}
\mid v(t) & -S_{\psi}^{\alpha}(t, 0) v(0)-\int_{0}^{t}(\psi(t)-\psi(s))^{\alpha-1} T_{\psi}^{\alpha}(t, s) f(s, v(s)) \psi^{\prime}(s) d s \mid \\
& \leq \int_{0}^{t}(\psi(t)-\psi(s))^{\alpha-1}\left\|T_{\psi}^{\alpha}(t, s)\right\| \varphi(s) \psi^{\prime}(s) d s \\
& \leq \lambda \varphi(t),
\end{aligned}
$$

for all $t \in[0, \infty)$. Let us denote by $u \in C([0, T], \infty)$ the unique mild solution of the Cauchy problem

$$
\left\{\begin{array}{l}
{ }_{0}^{C} D_{\psi}^{\alpha} u(t)=\mathcal{A} u(t)+f(t, u(t)), \quad t \in(0, \infty), \\
u(0)=v(0) .
\end{array}\right.
$$

We have

$$
u(t)=S_{\psi}^{\alpha}(t, 0) v(0)+\int_{0}^{t}(\psi(t)-\psi(s))^{\alpha-1} T_{\psi}^{\alpha}(t, s) f(s, u(s)) \psi^{\prime}(s) d s, \quad t \in[0, \infty) .
$$

It follows that

$$
\begin{aligned}
|v(t)-u(t)| \leq & \left|v(t)-S_{\psi}^{\alpha}(t, 0) v(0)-\int_{0}^{t}(\psi(t)-\psi(s))^{\alpha-1} T_{\psi}^{\alpha}(t, s) f(s, u(s)) \psi^{\prime}(s) d s\right| \\
\leq & \left|v(t)-S_{\psi}^{\alpha}(t, 0) v(0)-\int_{0}^{t}(\psi(t)-\psi(s))^{\alpha-1} T_{\psi}^{\alpha}(t, s) f(s, v(s)) \psi^{\prime}(s) d s\right| \\
& +\mid \int_{0}^{t}(\psi(t)-\psi(s))^{\alpha-1} T_{\psi}^{\alpha}(t, s) f(s, v(s)) \psi^{\prime}(s) d s \\
& -\int_{0}^{t}(\psi(t)-\psi(s))^{\alpha-1} T_{\psi}^{\alpha}(t, s) f(s, u(s)) \psi^{\prime}(s) d s \mid \\
\leq & \left|v(t)-S_{\psi}^{\alpha}(t, 0) v(0)-\int_{0}^{t}(\psi(t)-\psi(s))^{\alpha-1} T_{\psi}^{\alpha}(t, s) f(s, v(s)) \psi^{\prime}(s) d s\right| \\
& +\int_{0}^{t}(\psi(t)-\psi(s))^{\alpha-1}\left\|T_{\psi}^{\alpha}(t, s)\right\||f(s, v(s))-f(s, u(s))| \psi^{\prime}(s) d s \\
\leq & \lambda \varphi(t)+\frac{\alpha M k(t)}{\Gamma(1+\alpha)} \int_{0}^{t}(\psi(t)-\psi(s))^{\alpha-1}|v(s)-u(s)| \psi^{\prime}(s) d s .
\end{aligned}
$$


By Corollary 2.12, we obtain

$$
|v(t)-u(t)| \leq \lambda \varphi(t) E_{\alpha}\left(M k(t)[\psi(t)-\psi(0)]^{\alpha}\right) .
$$

The proof is complete.

\section{Examples}

In this section, we give examples of fractional differential equation of compact and noncompact semigroup cases. The main results can be applied for a larger class of Caputo fractional derivative with respect to $\psi$. In particular, our results can be reduced to the examples in $[25,32]$ when $\psi(t)=t$.

Example 7.1 Let $E=L^{2}([0, \pi])$ equipped with the norm and inner product defined, respectively, for all $u, v \in L^{2}([0, \pi])$ by

$$
\|u\|=\left(\int_{0}^{\pi}|u(x)|^{2} d x\right)^{\frac{1}{2}} \quad \text { and } \quad\langle u, v\rangle=\int_{0}^{\pi} u(x) \overline{v(x)} d x
$$

Consider the following initial-boundary value problem of time-fractional parabolic partial differential equation with nonlinear source term:

$$
\left\{\begin{array}{l}
{ }_{0}^{C} D_{\psi}^{\alpha} u(x, t)-\frac{\partial^{2}}{\partial x^{2}} u(x, t)=f(t, u(x, t)), \quad t \in(0,1], x \in[0, \pi] \\
u(0, t)=u(\pi, t)=0, \quad t \in(0,1] \\
u(x, 0)=u_{0}(x), \quad x \in[0, \pi]
\end{array}\right.
$$

where $\alpha=\frac{2}{3}, \psi(t)=\sqrt{t+1}$ and $f(t, u)=\frac{1}{3} e^{-t} u(x, t)+t^{2 \alpha} \sin (u(x, t))$. We define an operator $\mathcal{A}: D(\mathcal{A}) \subset E \rightarrow E$ by

$$
D(\mathcal{A}):=\left\{v \in E: v, v^{\prime} \text { are absolutely continuous and } v^{\prime \prime} \in E, v(0)=v(\pi)=0\right\}
$$

and

$$
\mathcal{A} u=\frac{\partial^{2}}{\partial x^{2}} u
$$

It is well known that $\mathcal{A}$ has a discrete spectrum, the eigenvalue are $-n^{2}, n \in \mathbb{N}$, with the corresponding normalized eigenvectors $e_{n}(z)=\sqrt{\frac{2}{\pi}} \sin (n z)$. Then

$$
\mathcal{A} x=\sum_{n=1}^{\infty}-n^{2}\left\langle x, e_{n}\right\rangle e_{n}, \quad x \in D(\mathcal{A})
$$

Furthermore, $\mathcal{A}$ generates a uniformly bounded analytic semigroup $\{T(t)\}_{t \geq 0}$ in $E$ and is given by

$$
T(t) x=\sum_{n=1}^{\infty} e^{-n^{2} t}\left\langle x, e_{n}\right\rangle e_{n}, \quad x \in E
$$


with $\|T(t)\| \leq e^{-t}$ for all $t \geq 0$. Hence, we take $M=1$ which implies that $\sup _{t \in[0, \infty)}\|T(t)\|=$ 1 and $\left(\mathrm{H}_{1}\right)$ are satisfies.

Then for $t \in[0,1]$ we have

$$
\begin{aligned}
& \|f(t, u)\| \leq \frac{1}{3} e^{-t}\|u\|+t^{2 \alpha} \pi, \\
& \sup _{\|u\| \leq r}\|f(t, u)\| \leq \frac{1}{3} e^{-t} r+t^{2 \alpha} \pi=: h_{r}(t), \\
& \limsup _{r \rightarrow \infty} \frac{\left\|h_{r}(t)\right\|_{L^{\infty}}}{r}=\frac{1}{3}=: L,
\end{aligned}
$$

and

$$
\left\|f\left(t, u_{1}\right)-f\left(t, u_{2}\right)\right\| \leq \frac{4}{3}\left\|u_{1}-u_{2}\right\| \quad \text { for } u_{1}, u_{2} \in \Omega_{r} .
$$

Therefore, $\left(\mathrm{H}_{2}\right)-\left(\mathrm{H}_{4}\right)$ are satisfied. This yields

$$
\frac{M L}{\Gamma(1+\alpha)}(\psi(T)-\psi(0))^{\alpha}=\frac{1}{3 \Gamma\left(\frac{5}{3}\right)}(\psi(1)-\psi(0))^{\frac{2}{3}} \approx 0.2052<1 .
$$

Hence, according to Theorems 4.1 and 4.3, the problem (20) has a unique mild solution on $[0,1]$.

Moreover, $\left(\mathrm{H}_{5}\right)$ is satisfied. Then, by Theorem 4.5, the problem (20) has a global mild solution $u \in C([0, \infty), E)$.

Example 7.2 Consider the following initial-boundary value problem of time-fractional parabolic partial differential equation with nonlinear source term:

$$
\left\{\begin{array}{l}
{ }_{0}^{\mathrm{C}} D_{\psi}^{\alpha} u(x, t)-\Delta u(x, t)=f(t, u(x, t)), \quad t \in(0,1], x \in[0,1] \\
u(0, t)=u(1, t)=0, \quad t \in[0,1] \\
u(x, 0)=u_{0}(x), \quad x \in[0,1]
\end{array}\right.
$$

where $\alpha=\frac{1}{2}, \psi(t)=e^{t}$ and $f(t, u)=\frac{1}{5} e^{-t}|u(x, t)|$. Let $E=L^{2}([0,1])$ and $\mathcal{A}: D(\mathcal{A}) \subset E \rightarrow E$ be an operator defined by

$$
D(\mathcal{A}):=H^{2}(0,1) \cap H_{0}^{1}(0,1)=\left\{v \in H^{2}(0,1): v(0)=v(1)=0\right\} \quad \text { and } \quad \mathcal{A} u=\Delta u,
$$

where $H^{2}(0,1)$ is the completion of the space $C^{2}(0,1)$ with respect to the norm

$$
\|u\|_{H^{2}(0,1)}=\left(\int_{0}^{1} \sum_{|\mu| \leq 2}\left|D^{\mu} u(x)\right|^{2} d x\right)^{\frac{1}{2}},
$$

and $C^{2}$ is the set of all is the set of all continuous defined on $(0,1)$ which have continuous partial derivatives of order less than or equal to 2 , and $H_{0}^{1}(0,1)$ is the completion of $C^{1}(0,1)$ with respect to the norm $\|u\|_{H^{1}(0,1)}$.

Then for $t \in[0,1]$ we have

$$
\|f(t, u)\| \leq \frac{1}{5} e^{-t}\|u\|
$$




$$
\begin{aligned}
& \sup _{\|u\| \leq r}\|f(t, u)\| \leq \frac{1}{5} e^{-t} r=: h_{r}(t), \\
& \limsup _{r \rightarrow \infty} \frac{\left\|h_{r}(t)\right\|_{L^{\infty}}}{r}=\frac{1}{2}, \\
& \left\|f\left(t, u_{1}\right)-f\left(t, u_{2}\right)\right\| \leq \frac{1}{5}\left\|u_{1}-u_{2}\right\| \quad \text { for } u_{1}, u_{2} \in \Omega_{r},
\end{aligned}
$$

and

$$
\mu(f(t, D)) \leq \frac{1}{5} \mu(D), \quad t \in[0,1] \text { and } D \in \Omega_{r} .
$$

Therefore, $\left(\mathrm{H}_{2}\right)-\left(\mathrm{H}_{3}\right)$ and $\left(\mathrm{H}_{6}\right)$ are satisfied. We take $M=1, L=\frac{1}{5}$ and $K=\frac{1}{5}$. This yields

$$
\frac{M L}{\Gamma(1+\alpha)}(\psi(T)-\psi(0))^{\alpha}=\frac{1}{25 \Gamma\left(\frac{3}{2}\right)}(\psi(1)-\psi(0))^{\frac{1}{2}} \approx 0.0592<1
$$

and

$$
\frac{4 M K}{\Gamma(1+\alpha)}(\psi(T)-\psi(0))^{\alpha}=\frac{4}{25 \Gamma\left(\frac{3}{2}\right)}(\psi(1)-\psi(0))^{\frac{1}{2}} \approx 0.2367<1 .
$$

Hence, according to Theorem 5.1 and 4.3, the problem (21) has unique mild solution on $[0,1]$.

Moreover, $\left(\mathrm{H}_{5}\right)$ is satisfied. Then Theorem 5.4 implies that the problem (21) has a global mild solution $u \in C([0, \infty), E)$.

\section{Conclusion}

We construct a mild solution for fractional evolution equation based on Laplace transform with respect to $\psi$-function. We obtain the local and global existence and uniqueness of mild solution for the problem with $\psi$-Caputo fractional derivative, which can be reduced to the classical Caputo fractional derivative in previous work. Furthermore, the form of a fundamental solution obtained in this work is a foundation result for further investigation such as the problem with perturbation, delay and a nonlocal term.

\section{Acknowledgements}

The authors would like to thank referees for valuable comments and suggestions to improve the manuscript. Moreover, the first author is grateful to the Research Professional Development Project Under the Science Achievement Scholarship of Thailand (SAST) for financial support.

Funding

The Science Achievement Scholarship of Thailand (SAST).

Availability of data and materials

The data that support the findings of this study are available from the authors, upon request.

Competing interests

The authors declare that they have no competing interests.

Authors' contributions

The main idea of this paper was proposed and mainly proved by PSN, while AS performed some proofs and provided some examples. All authors read and approved the final manuscript.

\section{Publisher's Note}

Springer Nature remains neutral with regard to jurisdictional claims in published maps and institutional affiliations. 


\section{References}

1. Oldham, K.B., Spanier, J.: The Fractional Calculus: Theory and Applications of Differentiation and Integration to Arbitrary Order. Mathematics in Science and Engineering. Academic Press, New York (1974)

2. Kilbas, A.A., Srivastava, H.M., Trujillo, J.J.: Theory and Applications of Fractional Differential Equations. Elsevier, Amsterdam (2006)

3. Anastassiou, G.A.: On right fractional calculus. Chaos Solitons Fractals 42(1), 365-376 (2009)

4. Atangana, A.: Convergence and stability analysis of a novel iteration method for fractional biological population equation. Neural Comput. Appl. 25(5), 1021-1030 (2014)

5. Gaul, L., Klein, P., Kemple, S.: Damping description involving fractional operators. Mech. Syst. Signal Process. 5(2), 81-88 (1991)

6. Glockle, W.G., Nonnenmacher, T.F.: A fractional calculus approach to self-similar protein dynamics. Biophys. J. 68(1), 46-53 (1995)

7. Metzler, R., Schick, W., Kilian, H.G., Nonnenmacher, T.F.: Relaxation in filled polymers: a fractional calculus approach. J. Chem. Phys. 103, 7180-7186 (1995)

8. Mainardi, F.: Fractional calculus: some basic problems in continuum and statistical mechanics. In: Fractals and Fractional Calculus in Continuum Mechanics, pp. 291-348. Springer, Wien (1997)

9. Hilfer, R.: Applications of Fractional Calculus in Physics. World Scientific, Singapore (2000)

10. Kumar, D., Singh, J., Baleanu, D.: On the analysis of vibration equation involving a fractional derivative with Mittag-Leffler law. Math. Methods Appl. Sci. 43(1), 443-457 (2020)

11. Baleanu, D., Asad, J.H., Jajarmi, A.: New aspects of the motion of a particle in a circular cavity. Proc. Rom. Acad., Ser. A: Math. Phys. Tech. Sci. Inf. Sci. 19(2), 361-367 (2018)

12. Baleanu, D., Jajarmi, A., Sajjadi, S.S., Mozyrska, D.: A new fractional model and optimal control of a tumor-immune surveillance with non-singular derivative operator. Chaos, Interdiscip. J. Nonlinear Sci. 29(8), 083127 (2019)

13. Baleanu, D., Asad, J.H., Jajarmi, A.: The fractional model of spring pendulum: new features within different kernels. Proc. Rom. Acad., Ser. A: Math. Phys. Tech. Sci. Inf. Sci. 19(3), 447-454 (2018)

14. Jajarmi, A., Baleanu, D., Sajjadi, S.S., Asad, J.H.: A new feature of the fractional Euler-Lagrange equations for a coupled oscillator using a nonsingular operator approach. Front. Phys. 7, 196 (2019)

15. Jajarmi, A., Arshad, S., Baleanu, D.: A new fractional modelling and control strategy for the outbreak of dengue fever. Phys. A, Stat. Mech. Appl. 535, 122524 (2019)

16. Yıldız, T.A., Jajarmi, A., Yıldız, B., Baleanu, D.: New aspects of time fractional optimal control problems within operators with nonsingular kernel. Discrete Contin. Dyn. Syst. 13(3), 407-428 (2020)

17. Jajarmi, A., Ghanbari, B., Baleanu, D.: A new and efficient numerical method for the fractional modelling and optimal control of diabetes and tuberculosis co-existence. Chaos, Interdiscip. J. Nonlinear Sci. 29(9), 093111 (2019)

18. Ziane, D., Cherif, M.H., Baleanu, D., Belghaba, K.: Exact solution for nonlinear local fractional partial differential equations. J. Appl. Comput. Mech. 6(2), 200-208 (2020)

19. Kumar, S., Kumar, A., Abbas, S., Qurashi, M.A., Baleanu, D.: A modified analytical approach with existence and uniqueness for fractional Cauchy reaction-diffusion equations. Adv. Differ. Equ. 2020(1), 28 (2020)

20. Mohammadi, F., Moradi, L., Baleanu, D., Jajarmi, A.: A hybrid functions numerical scheme for fractional optimal control problems: application to nonanalytic dynamic systems. J. Vib. Control 24(21), 5030-5043 (2018)

21. Cădariu, L:: Stabilitatea Ulam-Hyers-Bourgin pentru ecuatii functionale. Ed. Univ. Vest Timişoara, Timişara (2007)

22. Hyers, D.H., Isac, G., Rassias, T.M.: Stability of Functional Equations in Several Variables. Birkhäuser, Basel (1998)

23. Jung, S.M.: Hyers-Ulam-Rassias Stability of Functional Equations in Mathematical Analysis. Hadronic Press, Palm Harbor (2001)

24. Wang, J., Zhou, Y.: A class of fractional evolution equations and optimal controls. Nonlinear Anal., Real World Appl. 12(1), 262-272 (2011)

25. Zhou, Y., Jiao, F.: Nonlocal Cauchy problem for fractional evolution equations. Nonlinear Anal., Real World Appl. 11(5), $4465-4475$ (2010)

26. Zhou, Y., Jiao, F.: Existence of mild solutions for fractional neutral evolution equations. Comput. Math. Appl. 59, 1063-1077 (2010)

27. El-Borai, M.M., El-Nadi, K.S., El-Akabawy, E.G.: On some fractional evolution equations. Comput. Math. Appl. 59(3), $1352-1355$ (2010)

28. Li, M., Chen, C., Li, F.B.: On fractional powers of generators of fractional resolvent families. J. Funct. Anal. 259(10), 2702-2726 (2010)

29. Li, K., Peng, J., Jia, J.: Cauchy problems for fractional differential equations with Riemann-Liouville fractional derivatives. J. Funct. Anal. 263(2), 476-510 (2012)

30. Shu, X., Shi, Y.: A study on the mild solution of impulsive fractional evolution equations. Appl. Math. Comput. 273, 465-476 (2016)

31. Wang, R.N., Chen, D.H., Xiao, T.J.: Abstract fractional Cauchy problems with almost sectorial operators. J. Differ. Equ. 252(1), 202-235 (2012)

32. Chen, P., Li, Y., Chen, Q., Feng, B.: On the initial value problem of fractional evolution equations with noncompact semigroup. Comput. Math. Appl. 67(5), 1108-1115 (2014)

33. El-Borai, M.M.: The fundamental solutions for fractional evolution equations of parabolic type. J. Appl. Math. Stoch. Anal. 3, 197-211 (2004)

34. Li, K., Peng, J.: Fractional resolvents and fractional evolution equations. Appl. Math. Lett. 25(5), 808-812 (2012)

35. Wang, J., Zhou, Y.: Mittag-Leffler-Ulam stabilities of fractional evolution equations. Appl. Math. Lett. 25(4), 723-728 (2012)

36. Hilfer, R., Luchko, Y., Tomovski, Z.: Operational method for the solution of fractional differential equations with generalized Riemann-Liouville fractional derivatives. Fract. Calc. Appl. Anal. 12(3), 299-318 (2009)

37. Diethelm, K.: The Analysis of Fractional Differential Equations: An Application-Oriented Exposition Using Differential Operators of Caputo Type. Springer, Heidelberg (2010)

38. Almeida, R.: A Caputo fractional derivative of a function with respect to another function. Commun. Nonlinear Sci. Numer. Simul. 44, 460-481 (2017) 
39. Jarad, F., Abdeljawad, T.: Generalized fractional derivatives and Laplace transform. Discrete Contin. Dyn. Syst., Ser. S 13(3), 709-722 (2019)

40. Samko, S.G., Kilbas, A.A., Marichev, O.I. Fractional Integrals and Derivatives: Theory and Applications. Gordon \& Breach, Yverdon (1993)

41. Adjabi, Y., Jarad, F., Abdeljawad, T.: On generalized fractional operators and a Gronwall type inequality with applications. Filomat 31(17), 5457-5473 (2017)

42. da Vanterler, J., Sousa, C., Capelas de Oliveira, E.: A Gronwall inequality and the Cauchy-type problem by means of $\psi$-Hilfer operator. Differ. Equ. Appl. 11(1), 87-106 (2019)

43. Mainardi, F.: On the initial value problem for the fractional diffusion-wave equation. In: Rionero, S., Ruggeri, T. (eds.) Waves and Stability in Continuous Media, pp. 246-251 (1994)

44. Podlubny, I.: Fractional Differential Equations: An Introduction to Fractional Derivatives, Fractional Differential Equations, to Methods of Their Solution and Some of Their Applications, vol. 198. Elsevier, Amsterdam (1998)

45. Deimling, K.: Nonlinear Functional Analysis. Springer, Berlin (1985)

46. Banaś, J., Goebel, K.: Measures of Noncompactness in Banach Spaces. Lecture Notes in Pure and Applied Mathematics, vol. 60. Dekker, New York (1980)

47. Li, Y.X.: The positive solutions of abstract semilinear evolution equations and their applications. Acta Math. Sin. 39(5), 666-672 (1996)

48. Guo, D.J., Lakshmikantham, V., Liu, X.Z.: Nonlinear Integral Equations in Abstract Spaces. Kluwer Academic, Dordrecht (1996)

49. Heinz, H.P.: On the behaviour of noncompactness with respect to differentiation and integration of vector valued functions. Nonlinear Anal. 7(12), 1351-1371 (1983)

50. Liu, L., Guo, F., Wu, C., Wu, Y.: Existence theorems of global solutions for nonlinear Volterra type integral equations in Banach spaces. J. Math. Anal. Appl. 309(2), 638-649 (2005)

51. Engel, K., Nagel, R.: One-Parameter Semigroups for Linear Evolution Equations. Springer, New York (2000)

52. Pazy, A.: Semigroups of Linear Operators and Applications to Partial Differential Equations. Springer, New York (1983)

53. Mainardi, F., Paraddisi, P., Gorenflo, R.: Probability distributions generated by fractional diffusion equations. In: Kertesz, J., Kondor, I. (eds.) Econophysics: An Emerging Science. Kluwer Academic, Dordrecht (2000)

\section{Submit your manuscript to a SpringerOpen ${ }^{\circ}$ journal and benefit from:}

- Convenient online submission

- Rigorous peer review

- Open access: articles freely available online

- High visibility within the field

- Retaining the copyright to your article

Submit your next manuscript at $\gg$ springeropen.com 